\title{
Avoiding harmful oscillations in a drillstring through dynamical analysis
}

\author{
Eva M. Navarro-López ${ }^{\mathrm{a}, *}$, Domingo Cortés ${ }^{\mathrm{b}}$ \\ ${ }^{a}$ Escuela Técnica Superior de Ingenieros Industriales, Universidad de Castilla-La Mancha, Avda. Camilo José Cela s/n, \\ Edificio Politécnico, Campus Universitario, 13071 Ciudad Real, Spain \\ ${ }^{\mathrm{b}}$ Centro de Investigación y Estudios Avanzados del IPN, Departamento de Ingeniería Eléctrica, Av. IPN, 2508, \\ Col. San Pedro Zacatenco, 07360 México, D.F., México
}

Received 28 November 2005; received in revised form 11 October 2006; accepted 27 June 2007

\begin{abstract}
Complexity of oilwell drillstrings makes unfeasible the direct application of control methodologies to suppress nondesired oscillations existing in these systems. A reasonable alternative is to develop operation recommendations and parameter selection methods to guide the driller to avoid such oscillations. In this paper, by using dynamical analysis tools, operation recommendations and the detection of safe drilling parameters in a conventional oilwell drillstring are presented. To this end, a more generic lumped-parameter model than those considered until now is proposed. Particularly, this model takes into account the fact that the drillstring length increases as drilling operation makes progress. The analysis of a sliding motion giving rise to self-excited bit stick-slip oscillations and bit sticking phenomena at the bottom-hole assembly is performed. Finally, the identification of key drilling parameters ranges for which non-desired torsional oscillations are present is carried out by studying Hopf bifurcations in the vicinity of the system equilibrium point when rotary velocities are greater than zero.
\end{abstract}

(C) 2007 Elsevier Ltd. All rights reserved.

\section{Introduction}

Oilwell drillstrings are mechanical systems which undergo complex dynamical phenomena, often involving non-desired oscillations. Three main types of oscillations are distinguished: torsional (e.g., stick-slip oscillations), axial (e.g., bit bouncing phenomenon) and lateral (e.g., whirl motion due the out-of-balance of the drillstring) [1-4]. These oscillations are a source of failures which reduce penetration rates and increase drilling operation costs. Stick-slip phenomenon appearing at the bottom-hole assembly (BHA) is particularly harmful for the drillstring and it is a major cause of drill pipes and bit failures, in addition to well bore instability problems. For this reason, this paper is focused on stick-slip oscillations. They cause the top of the drillstring to rotate with a constant rotary speed, whereas the bit (cutting device) rotary speed varies between zero and up to six times the rotary speed measured at the surface.

\footnotetext{
*Corresponding author. Tel.: + 34667927042 ; fax: + 34926295361.

E-mail addresses: navarro.lopez@gmail.com (E.M. Navarro-López), domingo.cortes@gmail.com (D. Cortés).
} 
To deal with the problem of stick-slip oscillations, two challenging problems have to be solved: developing models to adequately describe the phenomenon and designing methodologies to help reduce its effects.

A model describing all drillstring dynamical phenomena would be too complex for analysis purposes. Simplifications are mandatory to keep equations manageable. Lumped-parameter models lead to more simple analysis and system simulation, in comparison to partial derivatives models. Several lumped-parameter models have been proposed in the literature to describe drillstring torsional behaviour. These models are of one degree [5,6] and two degrees of freedom [1,2,7-10]. Such models fail to reflect two important characteristics: (i) the fact that the drillstring length increases as drilling operation makes progress, (ii) oscillations appearing along the connected drill pipes and the drill collars (just above the bit). In this work, an $n$-dimensional lumped-parameter discontinuous model which overcomes such disadvantages is proposed. The discontinuity is introduced by the bit-rock interaction which is modelled by means of a dry friction combined with an exponential-decaying law.

Due to the complexity of the system and variables involved, the direct application of control methodologies in order to suppress non-desired oscillations phenomena can be unfeasible. It seems to be more reasonable to propose operation recommendations and drilling parameters selection methodologies in order to suppress oscillations and guide the driller. Following this approach, in this work, an analysis of system complex phenomena and bifurcations is performed. The existence of a sliding motion is proven. This motion is shown to be related to bit stick-slip oscillations and bit sticking phenomena. Transitions between several bit dynamics are identified. These transitions are shown to depend on the weight on the bit (WOB) and the torque given by the surface motor. It is shown that the presence of stick-slip and bit sticking phenomena is characterized by the dynamics of the drill collars (elements just above the bit) if an $n$-dimensional model is used, or by means of the top-rotary mechanism dynamics, if only two degrees of freedom are considered.

Local Poincaré-Andronov-Hopf bifurcations (often referred to as Hopf bifurcations) of the system equilibrium point when rotary velocities are greater than zero are also studied. Hopf bifurcations imply the birth, or the death, of a periodic orbit through a change in the stability of an equilibrium point and are the typical way in which instability arises in physical systems. Changes in drillstring behaviour are studied through variations in three parameters: (i) the weight on the bit, (ii) the rotary speed at the top-rotary drillstring system, (iii) the torque given by the surface motor $(u)$. Practical experience has shown them to be important for the drillstring behaviour [11]. Drillers employ these parameters in optimization drilling models [12]. These models are used to optimize the rate of penetration and drilling costs. However, the rotary speed and the weight on the bit are considered constant and no study of their influence on drillstring oscillations is usually made.

The intersection of the region of parameters where no Hopf bifurcations are present with the region where no stuck bit is possible provides a good estimation for parameters weight on the bit, $u$ and angular velocities to have safe drilling operations.

The paper is organized as follows. Section 2 presents an $n$-dimensional lumped-parameter model of the torsional behaviour of the drillstring including the bit-rock interaction. This model properly describes bit stick-slip oscillations and other non-desired bit phenomena. In Section 3, the effects of the weight on the bit and the torque $u$ on the bit behaviour are analysed. For this purpose, the existence of a sliding mode which causes different bit sticking phenomena is shown. Section 4 identifies the region of rotary velocities and weight on the bit in which non-desired oscillations are present. This is done by means of studying local bifurcations of the system equilibrium point when rotary velocities greater than zero are considered. Conclusions are given in the last section.

\section{A dynamic torsional model of a conventional drillstring}

The model presented in this section includes and generalizes other drillstring torsional lumped-parameter models appeared in the literature [1,2,5-10].

A conventional drillstring consists of the bottom-hole assembly and drill pipes screwed end to end to each other to form a long pipe (see Fig. 1). The bottom-hole assembly comprises the cutting device, referred to as 

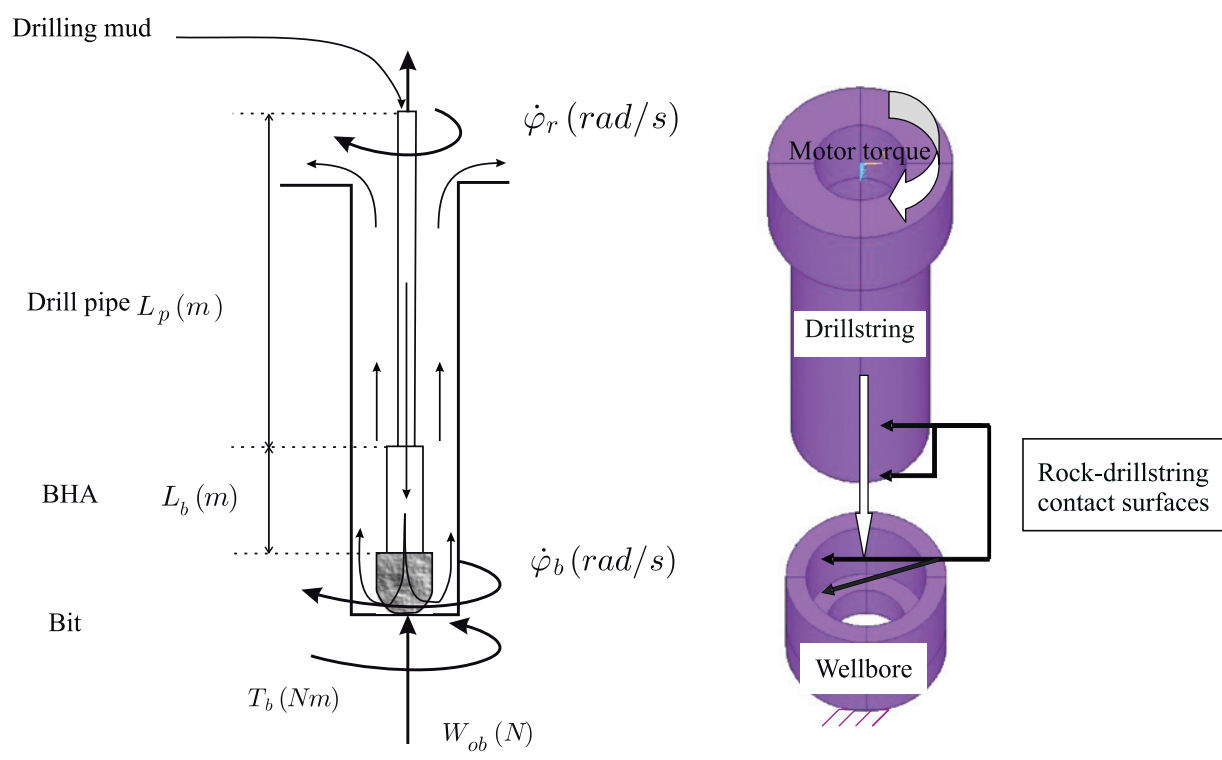

Fig. 1. Important elements in a conventional vertical drillstring. $\dot{\varphi}_{r}(\mathrm{rad} / \mathrm{s})$ top angular velocity, $\dot{\varphi}_{b}(\mathrm{rad} / \mathrm{s})$ bit angular velocity, $L_{p}(\mathrm{~m})$ drill pipe line length, $L_{b}(\mathrm{~m})$ bottom-hole assembly length, $W_{o b}(\mathrm{~N})$ weight on the bit, $T_{b}(\mathrm{Nm})$ torque on the bit.

bit, stabilizers (at least two spaced apart) which prevent the drillstring from underbalancing, and a series of pipe sections which are relatively heavy, known as drill collars. Drillstrings usually include a heavy-weight drill pipe at the top of the bottom-hole assembly. While the length of the bottom-hole assembly $\left(L_{b}\right)$ remains constant, the total length of the drill pipe line $\left(L_{p}\right)$ increases as the borehole depth does so and can reach several kilometers. An important element in the drilling is the drilling mud or fluid which, among others, has the function of cleaning, cooling and lubricating the bit. The drillstring is rotated from the surface by an electrical motor. The rotating mechanism can be of two types: a rotary table or a top drive.

Fig. 2 depicts a simplified torsional model of a conventional drillstring. It reflects the fact that the length of the drillstring (number of drill pipes) increases while the drilling operation advances. Four kinds of elements are considered: (i) the top-rotary system, (ii) $p$ drill pipes modelled as linear springs of torsional stiffness $k_{t}$ and torsional damping $c_{t}$, (iii) the bottom-hole assembly (including the drill collars), (iv) the bit. The drill pipes are connected to the inertias $J_{r}$ and $J$, corresponding to the inertia of the top-rotary system and to the inertia of each drill pipe. The number of drill pipes can be modified depending on system analysis requirements. The $p$ th drill pipe is connected to the drill collars $\left(J_{l}\right)$ by means of $k_{t l}$ and $c_{t l}$. Finally, the drill collars are connected to the bit $\left(J_{b}\right)$ by means of $k_{t b}$ and $c_{t b}$. At the bit, a viscous damping torque and a dry friction torque are taken into account. A viscous damping torque is also considered at the top-drive system.

The following assumptions are made: (i) the borehole and the drillstring are both vertical and straight, (ii) no lateral bit motion is present, (iii) the friction in the pipe connections and between the pipes and the borehole are neglected, (iv) the drilling mud is simplified by a viscous-type friction element at the bit, (v) the drilling mud fluids orbital motion is considered to be laminar, i.e., without turbulences, (vi) the motor dynamics is not considered, the drive torque is supposed to be constant and positive, (vii) the drill pipes are considered to have the same inertia. Under these assumptions and according to Fig. 2, the drillstring torsional model takes the following form:

$$
\begin{aligned}
& \ddot{\varphi}_{r}=-\frac{c_{t}}{J_{r}}\left(\dot{\varphi}_{r}-\dot{\varphi}_{1}\right)-\frac{k_{t}}{J_{r}}\left(\varphi_{r}-\varphi_{1}\right)+\frac{T_{m}-T_{a_{r}}\left(\dot{\varphi}_{r}\right)}{J_{r}}, \\
& \ddot{\varphi}_{1}=-\frac{c_{t}}{J}\left(2 \dot{\varphi}_{1}-\dot{\varphi}_{r}-\dot{\varphi}_{2}\right)-\frac{k_{t}}{J}\left(2 \varphi_{1}-\varphi_{r}-\varphi_{2}\right),
\end{aligned}
$$




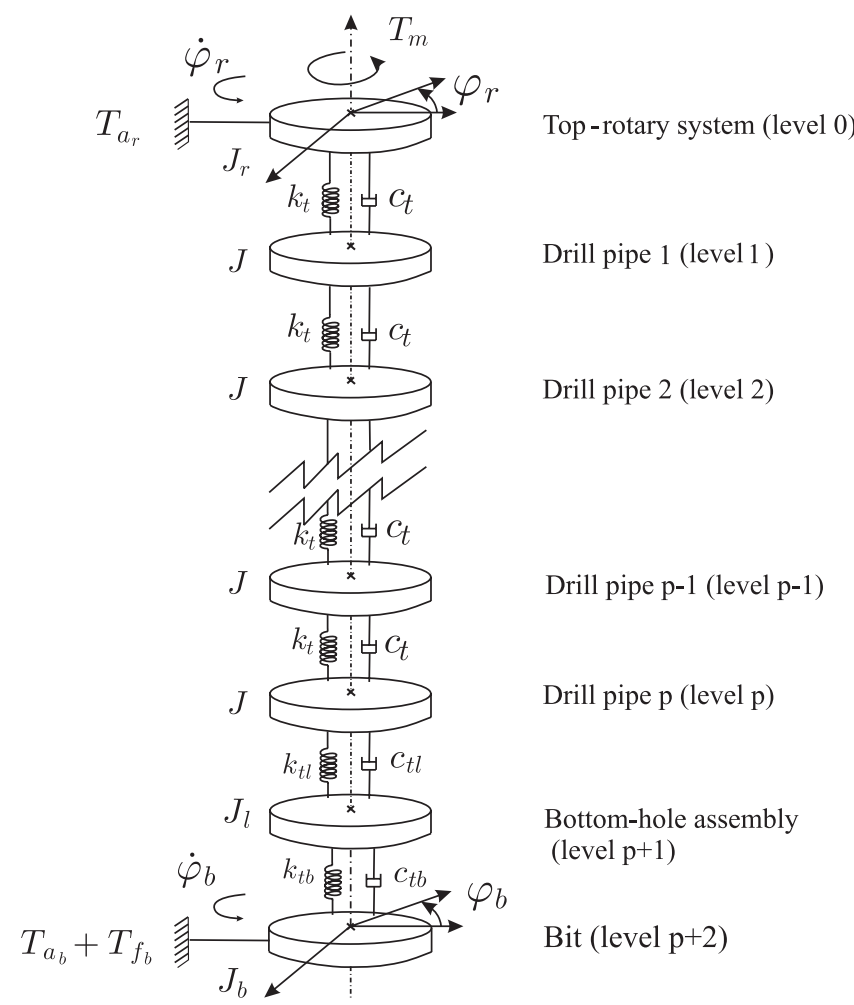

Fig. 2. Mechanical model describing the torsional behaviour of a conventional drillstring. All functions and parameters are explained in the text.

$$
\begin{aligned}
& \ddot{\varphi}_{j}=-\frac{c_{t}}{J}\left(2 \dot{\varphi}_{j}-\dot{\varphi}_{j+1}-\dot{\varphi}_{j-1}\right)-\frac{k_{t}}{J}\left(2 \varphi_{j}-\varphi_{j+1}-\varphi_{j-1}\right), \\
& \vdots \\
& \ddot{\varphi}_{p}=-\frac{c_{t}}{J}\left(\dot{\varphi}_{p}-\dot{\varphi}_{p-1}\right)-\frac{k_{t}}{J}\left(\varphi_{p}-\varphi_{p-1}\right)-\frac{c_{t l}}{J}\left(\dot{\varphi}_{p}-\dot{\varphi}_{l}\right)-\frac{k_{t l}}{J}\left(\varphi_{p}-\varphi_{l}\right), \\
& \ddot{\varphi}_{l}=-\frac{c_{t l}}{J_{l}}\left(\dot{\varphi}_{l}-\dot{\varphi}_{p}\right)-\frac{k_{t l}}{J_{l}}\left(\varphi_{l}-\varphi_{p}\right)-\frac{c_{t b}}{J_{l}}\left(\dot{\varphi}_{l}-\dot{\varphi}_{b}\right)-\frac{k_{t b}}{J_{l}}\left(\varphi_{l}-\varphi_{b}\right), \\
& \ddot{\varphi}_{b}=-\frac{c_{t b}}{J_{b}}\left(\dot{\varphi}_{b}-\dot{\varphi}_{l}\right)-\frac{k_{t b}}{J_{b}}\left(\varphi_{b}-\varphi_{l}\right)-\frac{T_{b}(\mathbf{x})}{J_{b}},
\end{aligned}
$$

with $p$ the number of drill pipes, $j=2, \ldots, p-1$. Eq. (1d) is used for the drill-pipe level $p$, i.e., for the inertia connected to $J_{l}$ through $k_{t l}$ and $c_{t l}, \varphi_{r}, \varphi_{p}, \varphi_{l}, \varphi_{b}$ are the angular displacements of the toprotary system, the drill pipes, the drill collars and the bit, respectively. $\dot{\varphi}_{r}, \dot{\varphi}_{p}, \dot{\varphi}_{l}, \dot{\varphi}_{b}$ are the angular velocities of the top-rotary system, the drill pipes, the drill collars and the bit, respectively. $T_{m}$ is the drive torque coming from the electrical motor at the surface. It is assumed that arbitrary torques can be applied without taking into account the actuator dynamics generating this torque, then $T_{m}=u$ with $u>0$ one of the system control inputs. $T_{a_{r}}$ is the viscous damping torque associated with $J_{r}$, it corresponds to the lubrication of the mechanical elements of the top-rotary system and $T_{a_{r}}=c_{r} \dot{\varphi}_{r}$, with $c_{r}$ the viscous damping coefficient. $T_{b}$ is the torque on the bit, $\mathbf{x}$ is the system state vector, and they are defined below. 
Let define,

$$
\begin{aligned}
\mathbf{x} & =\left(\varphi_{r}, \dot{\varphi}_{r}, \ldots, \varphi_{i}, \dot{\varphi}_{i}, \ldots, \varphi_{l}, \dot{\varphi}_{l}, \varphi_{b}, \dot{\varphi}_{b}\right)^{\mathrm{T}} \\
& =\left(x_{1}, x_{2}, \ldots, x_{2(i+1)-1}, x_{2(i+1)}, \ldots, x_{n-3}, x_{n-2}, x_{n-1}, x_{n}\right)^{\mathrm{T}},
\end{aligned}
$$

as the system state vector, with $i=1, \ldots, p$ and $n$ the order of the system or number of system variables. Note that $n$ is an even integer. Then, the system in Eq. (1) can be written as:

$$
\dot{\mathbf{x}}(t)=\mathbf{A x}(t)+\mathbf{B} u+\mathbf{T}_{\mathbf{f}}(\mathbf{x}(t)),
$$

where $\mathbf{B}$ and $\mathbf{T}_{\mathbf{f}}$ are given by

$$
\mathbf{B}=\left(\begin{array}{c}
0 \\
\frac{1}{J_{r}} \\
0 \\
\vdots \\
0
\end{array}\right), \quad \mathbf{T}_{\mathbf{f}}(\mathbf{x})=\left(\begin{array}{c}
0 \\
0 \\
\vdots \\
0 \\
-\frac{T_{f_{b}}(\mathbf{x})}{J_{b}}
\end{array}\right)
$$

and depending on the system dimension, $\mathbf{A}$ has the following form:

- For $n=4$. The system consists of two inertias $\left(J_{r}\right.$ and $\left.J_{b}\right)$ connected through $k_{t}$ and $c_{t}$. The system with $n=4$ is the most common drillstring model appeared in the literature. In this case,

$$
\mathbf{A}=\left(\begin{array}{cccc}
0 & 1 & 0 & 0 \\
-\frac{k_{t}}{J_{r}} & -\frac{c_{t}+c_{r}}{J_{r}} & \frac{k_{t}}{J_{r}} & \frac{c_{t}}{J_{r}} \\
0 & 0 & 0 & 1 \\
\frac{k_{t}}{J_{b}} & \frac{c_{t}}{J_{b}} & -\frac{k_{t}}{J_{b}} & -\frac{c_{t}+c_{b}}{J_{b}}
\end{array}\right)
$$

- For $n=6$. Three inertias are considered. $J_{r}$ is connected to $J_{l}$ through $k_{t}$ and $c_{t}$, and $J_{l}$ to $J_{b}$ through $k_{t b}$ and $c_{t b}$. In this case,

$$
\mathbf{A}=\left(\begin{array}{cccccc}
0 & 1 & 0 & 0 & 0 & 0 \\
-\frac{k_{t}}{J_{r}} & -\frac{c_{t}+c_{r}}{J_{r}} & \frac{k_{t}}{J_{r}} & \frac{c_{t}}{J_{r}} & 0 & 0 \\
0 & 0 & 0 & 1 & 0 & 0 \\
\frac{k_{t}}{J_{l}} & \frac{c_{t}}{J_{l}} & -\frac{k_{t}+k_{t b}}{J_{l}} & -\frac{c_{t}+c_{t b}}{J_{l}} & \frac{k_{t b}}{J_{l}} & \frac{c_{t b}}{J_{l}} \\
0 & 0 & 0 & 0 & 0 & 1 \\
0 & 0 & \frac{k_{t b}}{J_{b}} & \frac{c_{t b}}{J_{b}} & -\frac{k_{t b}}{J_{b}} & -\frac{c_{t b}+c_{b}}{J_{b}}
\end{array}\right)
$$


- For $n \geqslant 8$, that is, $p \geqslant 1$. The inertia $J_{l}$ is connected to its previous element by $k_{t l}$ and $c_{t l}$. In this case,

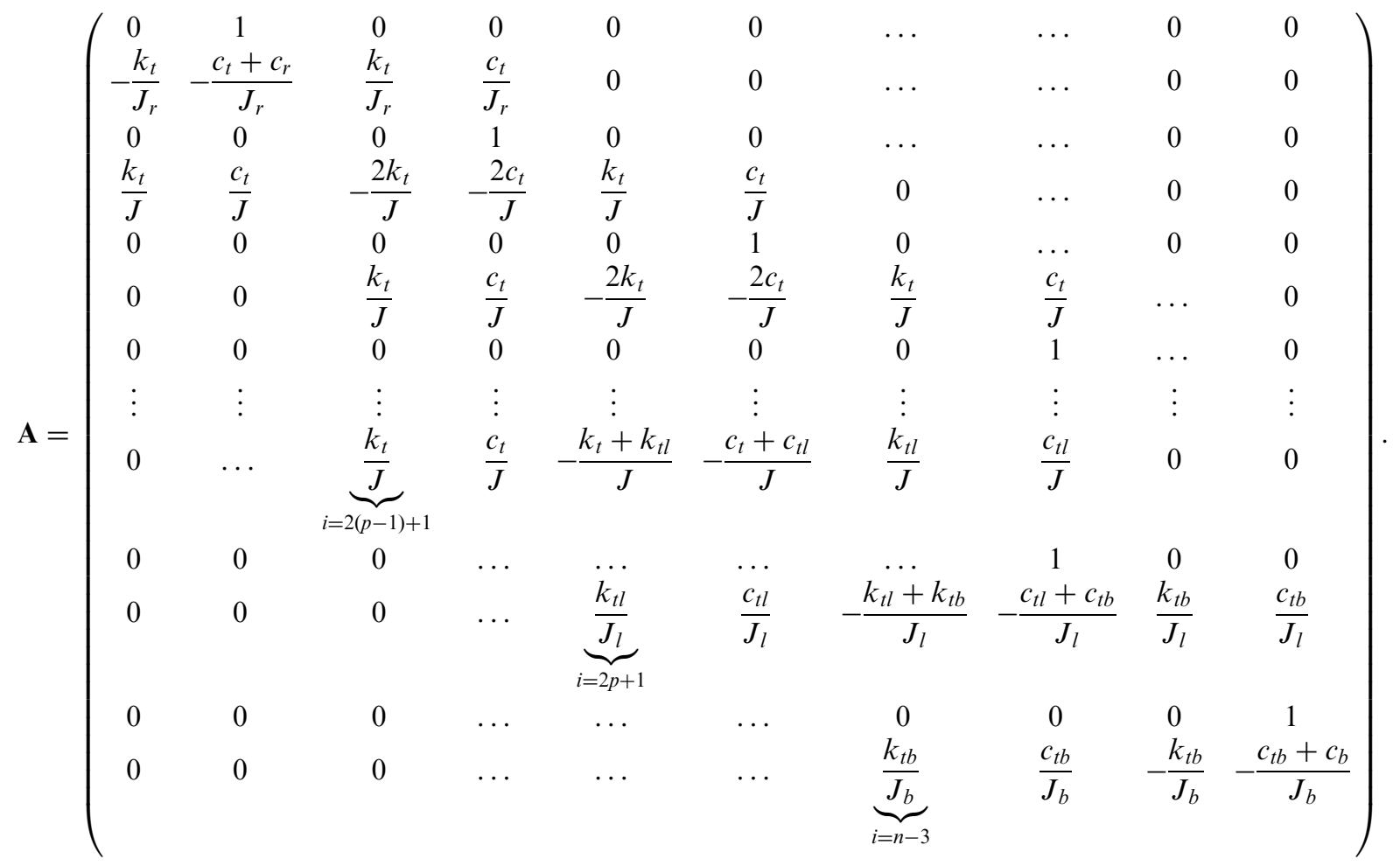

$T_{b}$ represents the dry friction torque plus the viscous damping torque associated with $J_{b}$, that is,

$$
T_{b}(\mathbf{x})=T_{a_{b}}\left(\dot{\varphi}_{b}\right)+T_{f_{b}}(\mathbf{x}),
$$

$T_{a_{b}}=c_{b} \dot{\varphi}_{b}$ is the viscous damping torque associated with the contact of the bit $\left(J_{b}\right)$ and the formation. This torque approximates the influence of the mud drilling on the bit behaviour. $T_{f_{b}}(\mathbf{x})$ is the friction that models the bit-rock contact and is now defined.

The bit-rock contact is proposed as a variation of the Stribeck friction together with the dry friction model [13]. The dry friction model when $\dot{\varphi}_{b}=0$, is approximated by a combination of the switch model proposed in Ref. [14] and the model in which a zero velocity band is introduced (Karnopp's model [15]). Thus,

$$
T_{f_{b}}(\mathbf{x})= \begin{cases}T_{e_{b}}(\mathbf{x}) & \text { if }\left|\dot{\varphi}_{b}\right|<D_{v},\left|T_{e_{b}}\right| \leqslant T_{s_{b}} \text { (stick), } \\ T_{s_{b}} \operatorname{sgn}\left(T_{e_{b}}(\mathbf{x})\right) & \text { if }\left|\dot{\varphi}_{b}\right|<D_{v},\left|T_{e_{b}}\right|>T_{s_{b}} \text { (stick-to-slip transition), } \\ f_{b}\left(\dot{\varphi}_{b}\right) \operatorname{sgn}\left(\dot{\varphi}_{b}\right) & \text { if }\left|\dot{\varphi}_{b}\right| \geqslant D_{v} \text { (sliding), }\end{cases}
$$

where $D_{v}>0, T_{e_{b}}$ is the reaction torque, that is, the torque that the static friction torque $T_{s_{b}}$ must overcome so that the bit moves, and $T_{s_{b}}=\mu_{s_{b}} W_{o b} R_{b}$, with $R_{b}>0$ the bit radius. The function $f_{b}\left(\dot{\varphi}_{b}\right)$ is given by,

$$
f_{b}\left(\dot{\varphi}_{b}\right)=R_{b} W_{o b} \mu_{b}\left(\dot{\varphi}_{b}\right),
$$

where $\mu_{b}\left(\dot{\varphi}_{b}\right)$ is the bit dry friction coefficient and $W_{o b}>0$ is the weight on the bit. $\mu_{b}\left(\dot{\varphi}_{b}\right)$ has the form,

$$
\mu_{b}\left(\dot{\varphi}_{b}\right)=\left[\mu_{c_{b}}+\left(\mu_{s_{b}}-\mu_{c_{b}}\right) \mathrm{e}^{-\gamma_{b} / v_{f}\left|\dot{\varphi}_{b}\right|}\right],
$$

where $\mu_{s_{b}}, \mu_{c_{b}}$ are the static and Coulomb friction coefficients associated with inertia $J_{b}$ and $0<\gamma_{b}<1$ is a constant defining the velocity decrease rate of $T_{f_{b}}$. The constant velocity $v_{f}$ is introduced in order 
(a)

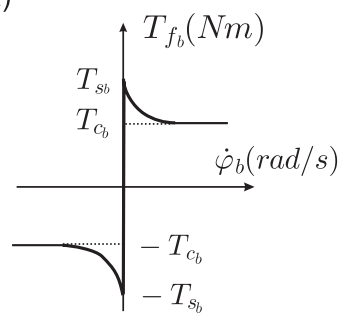

(b)

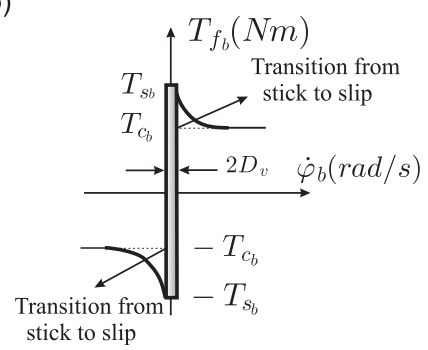

Fig. 3. Friction at the bit $\left(T_{f_{b}}\right)$ : (a) dry friction with an exponential-decaying law at the sliding phase, (b) switch friction model with a variation of Karnopp's friction model. $\dot{\varphi}_{b}(\mathrm{rad} / \mathrm{s})$ bit angular velocity, $T_{s_{b}}=\mu_{s_{b}} W_{o b} R_{b}(\mathrm{Nm})$ static friction torque, $T_{c_{b}}=\mu_{c_{b}} W_{o b} R_{b}(\mathrm{Nm})$ Coulomb friction torque, $D_{v}>0$.

to have appropriate units. Note that $\mu_{b}\left(\dot{\varphi}_{b}\right), \mu_{s_{b}}, \mu_{c_{b}} \in(0,1)$ and $\mu_{s_{b}}>\mu_{c_{b}}$. The reaction torque $T_{e_{b}}$ is given by

$$
T_{e_{b}}(\mathbf{x})= \begin{cases}c_{t}\left(\dot{\varphi}_{r}-\dot{\varphi}_{b}\right)+k_{t}\left(\varphi_{r}-\varphi_{b}\right)-T_{a_{b}}\left(\dot{\varphi}_{b}\right) & \text { if } n=4, \\ c_{t b}\left(\dot{\varphi}_{l}-\dot{\varphi}_{b}\right)+k_{t b}\left(\varphi_{l}-\varphi_{b}\right)-T_{a_{b}}\left(\dot{\varphi}_{b}\right) & \text { if } n>4 .\end{cases}
$$

The model in Eq. (9) depicted in Fig. 3(b) will be used for simulations. However, for the sake of simplicity, the function

$$
T_{f_{b}}\left(\dot{\varphi}_{b}\right)=R_{b} W_{o b}\left[\mu_{c_{b}}+\left(\mu_{s_{b}}-\mu_{c_{b}}\right) \mathrm{e}^{-\gamma_{b} / v_{f}\left|\dot{\varphi}_{b}\right|}\right] \operatorname{sgn}\left(\dot{\varphi}_{b}\right)
$$

depicted in Fig. 3(a) will be used for analysis purposes.

The exponential decaying behaviour of the torque-on-bit $T_{b}$ coincides with experimental bit torque values and is inspired in the models given in Refs. $[1,7,16]$.

The model in Eq. (3) with Eq. (13) is an $n$-dimensional discontinuous nonlinear system. The discontinuity introduced by the bit-rock friction causes different system complex dynamical phenomena.

\section{Effects of the WOB and the torque $u$ on stick-slip and other bit phenomena. Sliding motion-based analysis}

The influence of the pair $\left(W_{o b}, u\right)$ on the existence of stick-slip phenomenon and other non-desired bit situations will be identified in this section. For this purpose, the existence of a sliding motion is shown, and it is related to the presence of bit sticking phenomena. The drillstring sliding motion means that the bit is stuck. The discontinuous model in Eq. (3) with Eq. (13) will be used.

An example of the stick-slip phenomenon appearing at the bit is shown in Fig. 4 for the system in Eqs. (3)-(7) with Eq. (13) with $n=8, W_{o b}=100 \mathrm{~N}, u=8.3 \mathrm{Nm}$. The oscillations obtained for the drill pipe and the drill collars associated with the stick-slip bit motion are in accordance with real drillstrings behaviour. The model parameters used for the simulations presented along this section correspond to a reduced-scale model:

$$
\begin{aligned}
& J_{r}=0.518 \mathrm{~kg} \mathrm{~m}^{2}, \quad J_{b}=0.0318 \mathrm{~kg} \mathrm{~m}^{2}, \quad c_{t}=0.0001 \mathrm{Nm} \mathrm{s} / \mathrm{rad}, \quad k_{t}=0.073 \mathrm{Nm} / \mathrm{rad}, \\
& c_{b}=0.01 \mathrm{Nm} \mathrm{s} / \mathrm{rad}, \quad c_{r}=0.18 \mathrm{Nm} \mathrm{s} / \mathrm{rad}, \quad k_{t b}=k_{t}, \quad c_{t b}=c_{t l}=c_{t}, \quad k_{t l}=k_{t}, \quad J_{l}=J_{b}, \\
& J=0.025 \mathrm{~kg} \mathrm{~m}^{2}, \quad \mu_{c_{b}}=0.5, \quad \mu_{s_{b}}=0.8, \quad R_{b}=0.1 \mathrm{~m}, \quad D_{v}=10^{-6}, \quad \gamma_{b}=0.9, \quad v_{f}=1 .
\end{aligned}
$$

\subsection{Sliding motion existence}

The system in Eq. (3) with Eq. (13) can be written as a switching dynamical system:

$$
\dot{\mathbf{x}}= \begin{cases}\mathbf{f}^{+}(\mathbf{x}, \boldsymbol{\mu}) & \text { if } \sigma(\mathbf{x})>0, \\ \mathbf{f}^{-}(\mathbf{x}, \boldsymbol{\mu}) & \text { if } \sigma(\mathbf{x})<0,\end{cases}
$$


(a)

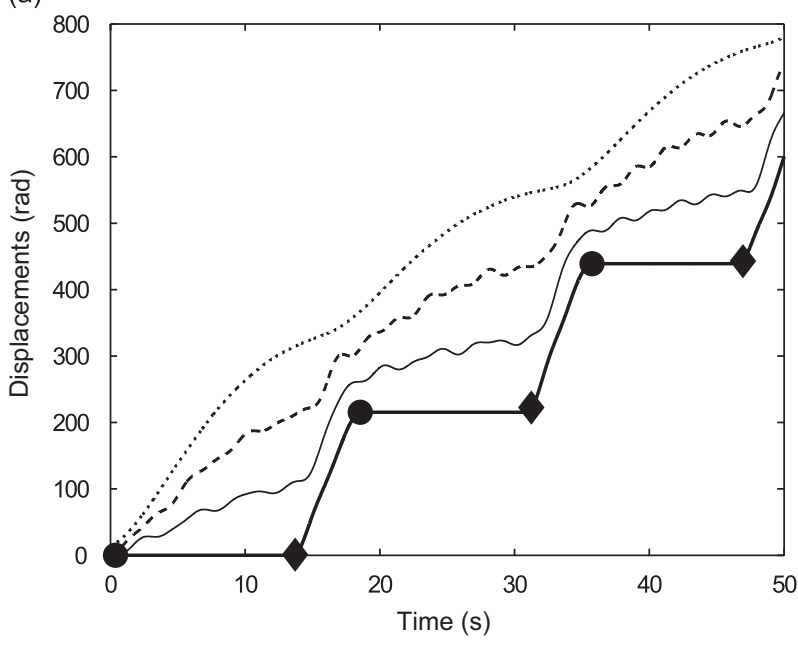

(b)

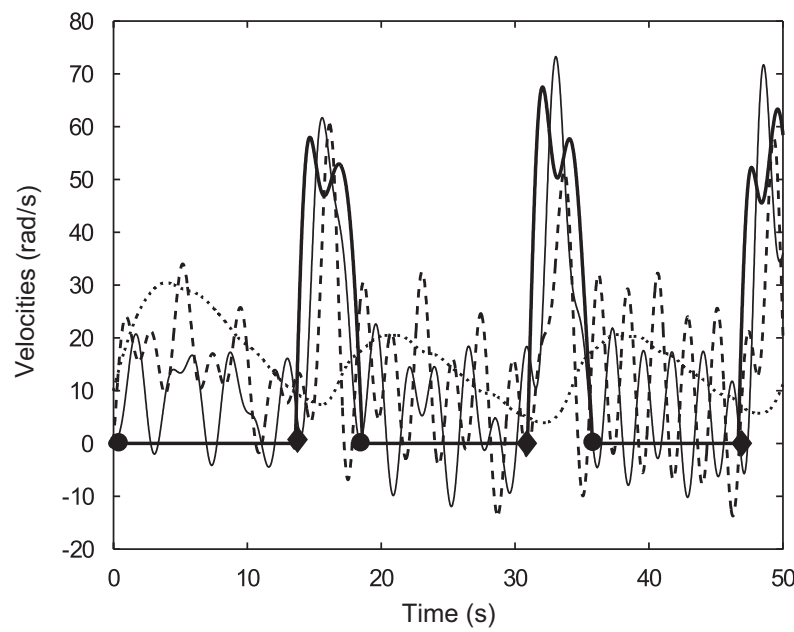

Fig. 4. Dynamics for the system with $n=8$ for the stick-slip situation: (a) angular displacements, (b) angular velocities. $\mathbf{x}_{\text {in }}(\bullet)$ and $\mathbf{x}_{\text {out }}(\boldsymbol{)})$ are the points at which the system trajectory enters and goes out of the sticking region. — bit angular displacement and velocity $\left(\varphi_{b}, \dot{\varphi}_{b}\right)$, - angular displacement and velocity of the drill collars $\left(\varphi_{l}, \dot{\varphi}_{l}\right),-$ - angular displacement and velocity of the drill pipe $\left(\varphi_{1}, \dot{\varphi}_{1}\right)$, $\cdots$ top-rotary system angular displacement and velocity $\left(\varphi_{r}, \dot{\varphi}_{r}\right)$.

where $\sigma(\mathbf{x})=x_{n}=\dot{\varphi}_{b}, \boldsymbol{\mu}=W_{o b} \times u \in \mathbb{R}^{2}$ is a parameter vector, $\mathbf{f}^{+}, \mathbf{f}^{-}: \mathbb{R}^{n+2} \rightarrow \mathbb{R}^{n}$ are smooth vector functions. $\mathbf{f}^{+}\left(\mathbf{x}, W_{o b}, u\right)=\mathbf{A} \mathbf{x}+\mathbf{B} u+\left.\mathbf{T}_{\mathbf{f}}(\mathbf{x})\right|_{T_{f_{b}}=T_{f_{b}}^{+}}$and $\mathbf{f}^{-}\left(\mathbf{x}, W_{o b}, u\right)=\mathbf{A x}+\mathbf{B} u+\left.\mathbf{T}_{\mathbf{f}}(\mathbf{x})\right|_{T_{f_{b}}=T_{f_{b}}^{-}}$. According to Eq. (13),

$$
\begin{aligned}
& T_{f_{b}}^{+}\left(\dot{\varphi}_{b}\right)=W_{o b} R_{b}\left[\mu_{c_{b}}+\left(\mu_{s_{b}}-\mu_{c_{b}}\right) \mathrm{e}^{-\left(\gamma_{b} / v_{f}\right) \dot{\varphi}_{b}}\right], \\
& T_{f_{b}}^{-}\left(\dot{\varphi}_{b}\right)=-W_{o b} R_{b}\left[\mu_{c_{b}}+\left(\mu_{s_{b}}-\mu_{c_{b}}\right) \mathrm{e}^{\left(\gamma_{b} / v_{f}\right) \dot{\varphi}_{b}}\right] .
\end{aligned}
$$

If the set

$$
\Sigma:=\left\{\mathbf{x} \in \mathbb{R}^{n}: \sigma(\mathbf{x})=0\right\},
$$

which is referred to as switching manifold, has a subset $\tilde{\Sigma} \subset \Sigma$ attractive from both sides, a sliding mode may occur [17]. If the system trajectory crosses the sliding region $\tilde{\Sigma}$, it will evolve within $\tilde{\Sigma}$ until it eventually reaches its boundary. The next proposition establishes that, in a certain state region, a sliding motion occurs for the system in Eq. (3) with Eq. (13).

Proposition 1. Consider a system in Eq. (3) with Eq. (13). Then, there is a set $\mathscr{S} \in \mathbb{R}^{n}$ in which the trajectories of the system go into a sliding motion on a subset of $\Sigma$.

Proof. Let denote $\dot{\sigma}=(\partial \sigma / \partial \mathbf{x}) \mathbf{f}\left(\mathbf{x}, W_{o b}, u\right)$ the time derivative of function $\sigma$ along the system trajectories. $\dot{\sigma}$ has the form:

$$
\dot{\sigma}=\dot{x}_{n}= \begin{cases}\frac{1}{J_{b}}\left[c_{t}\left(x_{2}-x_{4}\right)+k_{t}\left(x_{1}-x_{3}\right)-c_{b} x_{4}-T_{f_{b}}\left(x_{4}\right)\right] & \text { if } n=4, \\ \frac{1}{J_{b}}\left[c_{t_{b}}\left(x_{n-2}-x_{n}\right)+k_{t b}\left(x_{n-3}-x_{n-1}\right)-c_{b} x_{n}-T_{f_{b}}\left(x_{n}\right)\right] & \text { if } n>4 .\end{cases}
$$

It will be shown that there is a subset $\mathscr{S}$ of $\mathbb{R}^{n}$ where $\dot{\sigma} \sigma<0$, that is, a sliding mode can exist [17]. For the sake of simplicity, only the case $n>4$ is taken into account.

Consider the two cases:

Case 1: $\sigma<0$. In this case, $x_{n}<0$ and $T_{f_{b}}=T_{f_{b}}^{-}$. Hence, $\dot{\sigma}>0$ in the set,

$$
\left\{\mathbf{x} \in \mathbb{R}^{n}:\left|c_{t_{b}} x_{n-2}+k_{t b}\left(x_{n-3}-x_{n-1}\right)\right|<\left(c_{t b}+c_{b}\right)\left|x_{n}\right|-T_{f_{b}}^{-}\right\}
$$


Case 2: $\sigma>0$. In this case, $x_{n}>0$ and $T_{f_{b}}=T_{f_{b}}^{+}$. Hence, $\dot{\sigma}<0$ in the set,

$$
\left\{\mathbf{x} \in \mathbb{R}^{n}:\left|c_{t_{b}} x_{n-2}+k_{t b}\left(x_{n-3}-x_{n-1}\right)\right|<\left(c_{t b}+c_{b}\right)\left|x_{n}\right|+T_{f_{b}}^{+}\right\} .
$$

Combining both cases, $\dot{\sigma} \sigma<0$ is met in the set,

$$
\begin{aligned}
\mathscr{S}= & \left\{\mathbf{x} \in \mathbb{R}^{n}:\left|c_{t_{b}} x_{n-2}+k_{t b}\left(x_{n-3}-x_{n-1}\right)\right|<\left(c_{t b}+c_{b}\right)\left|x_{n}\right|\right. \\
& \left.+R_{b} W_{o b}\left[\mu_{c_{b}}+\left(\mu_{s_{b}}-\mu_{c_{b}}\right) \mathrm{e}^{-\left(\gamma_{b} / v_{f}\right)\left|x_{n}\right|}\right]\right\} .
\end{aligned}
$$

Since $\dot{\sigma} \sigma<0$ in the set $\mathscr{S}$, a sliding motion takes place when the system trajectory hits $\Sigma$ within this set.

From the form of $\mathscr{S}$ in Eq. (21), the sliding region $\tilde{\Sigma} \subset \Sigma$ is obtained as the set,

$$
\tilde{\Sigma}= \begin{cases}\left\{\mathbf{x} \in \Sigma:\left|k_{t}\left(\varphi_{r}-\varphi_{b}\right)+c_{t} \dot{\varphi}_{r}\right|<\mu_{s_{b}} W_{o b} R_{b}\right\} & \text { if } n=4, \\ \left\{\mathbf{x} \in \Sigma:\left|k_{t b}\left(\varphi_{l}-\varphi_{b}\right)+c_{t b} \dot{\varphi}_{l}\right|<\mu_{s_{b}} W_{o b} R_{b}\right\} & \text { if } n>4 .\end{cases}
$$

Let $\partial \tilde{\Sigma}$ denote the boundary of $\tilde{\Sigma}$, and $\partial \tilde{\Sigma}=\partial \tilde{\Sigma}^{+} \cup \partial \tilde{\Sigma}^{-}$with:

$$
\begin{aligned}
& \partial \tilde{\Sigma}^{+}=\left\{\mathbf{x} \in \Sigma: \frac{\partial \sigma}{\partial \mathbf{x}} \mathbf{f}^{+}\left(\mathbf{x}, W_{o b}, u\right)=0\right\}, \\
& \partial \tilde{\Sigma}^{-}=\left\{\mathbf{x} \in \Sigma: \frac{\partial \sigma}{\partial \mathbf{x}} \mathbf{f}^{-}\left(\mathbf{x}, W_{o b}, u\right)=0\right\} .
\end{aligned}
$$

For the system in Eq. (3) with Eq. (13), it is obtained,

- For $n=4$ :

$$
\begin{aligned}
& \partial \tilde{\Sigma}^{+}=\left\{\mathbf{x} \in \Sigma: k_{t}\left(\varphi_{r}-\varphi_{b}\right)+c_{t} \dot{\varphi}_{r}=\mu_{s_{b}} W_{o b} R_{b}\right\}, \\
& \partial \tilde{\Sigma}^{-}=\left\{\mathbf{x} \in \Sigma: k_{t}\left(\varphi_{r}-\varphi_{b}\right)+c_{t} \dot{\varphi}_{r}=-\mu_{s_{b}} W_{o b} R_{b}\right\}
\end{aligned}
$$

- For $n>4$ :

$$
\begin{aligned}
& \partial \tilde{\Sigma}^{+}=\left\{\mathbf{x} \in \Sigma: k_{t b}\left(\varphi_{l}-\varphi_{b}\right)+c_{t b} \dot{\varphi}_{l}=\mu_{s_{b}} W_{o b} R_{b}\right\}, \\
& \partial \tilde{\Sigma}^{-}=\left\{\mathbf{x} \in \Sigma: k_{t b}\left(\varphi_{l}-\varphi_{b}\right)+c_{t b} \dot{\varphi}_{l}=-\mu_{s_{b}} W_{o b} R_{b}\right\} .
\end{aligned}
$$

The sliding motion can be described by $\dot{\mathbf{x}}=\mathbf{f}_{\mathbf{s}}\left(\mathbf{x}, W_{o b}, u\right)$, where $\mathbf{f}_{\mathbf{s}}$ is a vector field tangent to $\Sigma$ for $\mathbf{x} \in \tilde{\Sigma}$. $\mathbf{f}_{\mathbf{s}}$ can be calculated by means of the equivalent control method [17,18]. For the drillstring model in Eq. (3) with Eq. (13), $T_{f_{b}}$ plays the role of the equivalent control and,

$$
\mathbf{f}_{\mathbf{s}}\left(\mathbf{x}, W_{o b}, u\right)=\mathbf{A x}+\mathbf{B} u+\left.\mathbf{T}_{\mathbf{f}}(\mathbf{x})\right|_{T_{f_{b}}=T_{f b e q}},
$$

where $T_{f b e q}$ is the solution for $T_{f_{b}}$ of equation $\dot{\sigma}=0$, that is,

$$
T_{f b e q}(\mathbf{x})= \begin{cases}k_{t}\left(\varphi_{r}-\varphi_{b}\right)+c_{t}\left(\dot{\varphi}_{r}-\dot{\varphi}_{b}\right)-c_{b} \dot{\varphi}_{b} & \text { if } n=4, \\ k_{t b}\left(\varphi_{l}-\varphi_{b}\right)+c_{t b}\left(\dot{\varphi}_{l}-\dot{\varphi}_{b}\right)-c_{b} \dot{\varphi}_{b} & \text { if } n>4 .\end{cases}
$$

The presence of a sliding mode makes necessary to distinguish different kinds of equilibrium points. The change of properties of these equilibria will define different types of system bifurcations, and consequently, different types of system behaviour which will be analysed in Section 3.2.

Definition 2 (Filippov [18], Cunha et al. [19]). $\overline{\mathbf{x}} \in \mathbb{R}^{n}$ is addressed as standard equilibrium point of the system in Eq. (15) if there exists $\overline{\boldsymbol{\mu}} \in \mathbb{R}^{2}$ such that $\mathbf{f}^{+}(\overline{\mathbf{x}}, \overline{\boldsymbol{\mu}})=0$ or $\mathbf{f}^{-}(\overline{\mathbf{x}}, \overline{\boldsymbol{\mu}})=0$. Let $\tilde{\mathbf{x}} \in \mathbb{R}^{n}, \tilde{\boldsymbol{\mu}} \in \mathbb{R}^{2}$ be such that $\mathbf{f}_{\mathbf{s}}(\tilde{\mathbf{x}}, \tilde{\boldsymbol{\mu}})=0$ and $\sigma(\tilde{\mathbf{x}})=0$. $\tilde{\mathbf{x}}$ is said to be a real quasi-equilibrium point if $\tilde{\mathbf{x}} \in \tilde{\Sigma}$. If $\tilde{\mathbf{x}} \notin \tilde{\Sigma}, \tilde{\mathbf{x}}$ is referred to as virtual quasi-equilibrium point. 
By making $\mathbf{f}_{\mathbf{s}}\left(\tilde{\mathbf{x}}, W_{o b}, u\right)=0$, the quasi-equilibrium points on $\Sigma$ can be calculated, and they have the following form for each time the system trajectory enters $\Sigma$ :

- For $n=4$ :

$$
\tilde{x}_{2}=\tilde{x}_{4}=0, \quad \tilde{x}_{1}=\frac{u}{k_{t}}+\bar{\varphi}_{b}
$$

- For $n=6$ :

$$
\begin{aligned}
& \tilde{x}_{2}=\tilde{x}_{4}=\tilde{x}_{6}=0, \\
& \tilde{x}_{1}=\left(\frac{1}{k_{t}}+\frac{1}{k_{t b}}\right) u+\bar{\varphi}_{b}, \\
& \tilde{x}_{3}=\frac{u}{k_{t b}}+\bar{\varphi}_{b} .
\end{aligned}
$$

- For $n>4$ :

$$
\begin{aligned}
& \tilde{x}_{2 i}=0, \quad i=1, \ldots, \frac{n}{2}, \\
& \tilde{x}_{n-3}=\tilde{\varphi}_{l}=\frac{u}{k_{t b}}+\bar{\varphi}_{b}, \\
& \tilde{x}_{n-5}=\tilde{\varphi}_{p}=\left(\frac{1}{k_{t b}}+\frac{1}{k_{t l}}\right) u+\bar{\varphi}_{b}, \\
& \tilde{x}_{2 j-1}=\left(\frac{p-j+1}{k_{t}}+\frac{1}{k_{t b}}+\frac{1}{k_{t l}}\right) u+\bar{\varphi}_{b}, \quad j=1, \ldots, p,
\end{aligned}
$$

where $\overline{\varphi_{b}}$ is the constant value of $\varphi_{b}$ during the time the trajectory of the system stays in $\Sigma$, this value varies every time the system trajectory enters again $\Sigma$. See Fig. 4(a).

Proposition 3. The quasi-equilibrium point $\tilde{x}$ given by Eqs. (28)-(30) is asymptotically stable.

Proof. In order to prove the stability of $\tilde{\mathbf{x}}$, the following Lyapunov function is considered, which corresponds to the sum of the kinetic and potential energy of the system on $\Sigma$ :

- For $n=4$ :

$$
V(\mathbf{x}, \tilde{\mathbf{x}})=\frac{1}{2}\left[k_{t}\left(x_{1}-\tilde{x}_{1}\right)^{2}+J_{r} x_{2}^{2}\right]
$$

- For $n=6$ :

$$
V(\mathbf{x}, \tilde{\mathbf{x}})=\frac{1}{2}\left\{k_{t}\left[\left(x_{1}-x_{3}\right)-\left(\tilde{x}_{1}-\tilde{x}_{3}\right)\right]^{2}+k_{t b}\left(x_{3}-\tilde{x}_{3}\right)^{2}+J_{r} x_{2}^{2}+J x_{4}^{2}\right\} .
$$

- For $n>6$ :

$$
\begin{aligned}
V(\mathbf{x}, \tilde{\mathbf{x}})= & \frac{1}{2} k_{t}\left\{\left[\left(x_{1}-x_{3}\right)-\left(\tilde{x}_{1}-\tilde{x}_{3}\right)\right]^{2}+\left[\left(x_{3}-x_{5}\right)-\left(\tilde{x}_{3}-\tilde{x}_{5}\right)\right]^{2}+\cdots\right. \\
& \left.+\left[\left(x_{n-7}-x_{n-5}\right)-\left(\tilde{x}_{n-7}-\tilde{x}_{n-5}\right)\right]^{2}\right\} \\
& +\frac{1}{2} k_{t l}\left[\left(x_{n-5}-x_{n-3}\right)-\left(\tilde{x}_{n-5}-\tilde{x}_{n-3}\right)\right]^{2}+\frac{1}{2} k_{t b}\left(x_{n-3}-\tilde{x}_{n-3}\right)^{2} \\
& +\frac{1}{2}\left[J_{r} x_{2}^{2}+J\left(x_{4}^{2}+\cdots+x_{n-4}^{2}\right)+J_{l} x_{n-2}^{2}\right],
\end{aligned}
$$


(a)

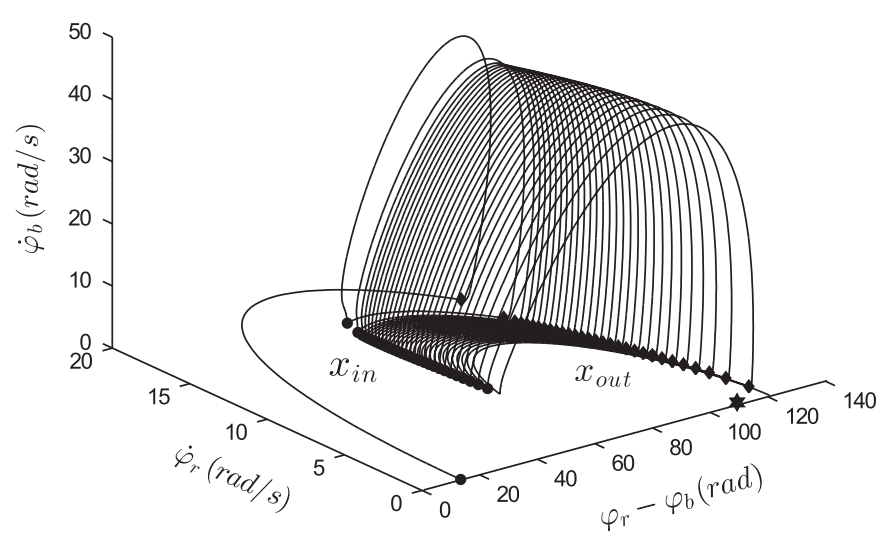

(b)

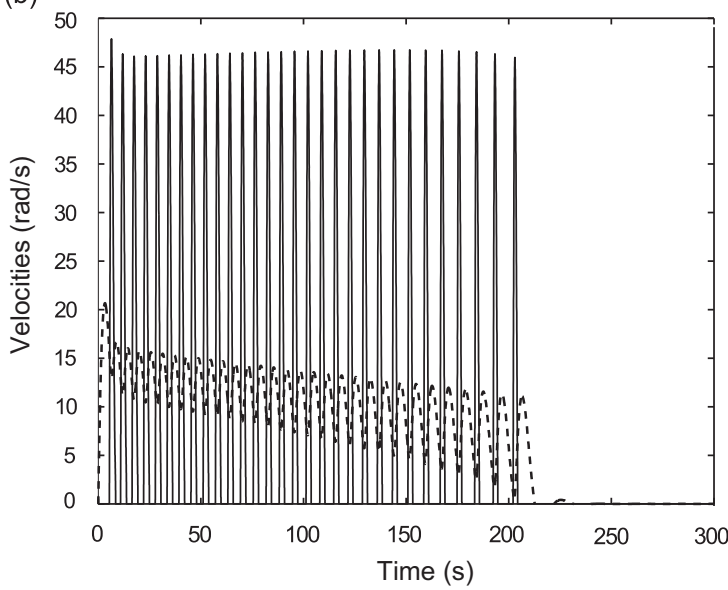

Fig. 5. Response of the system with $n=4$ for a varying $W_{o b}$ from $90 \mathrm{~N}$ to $150 \mathrm{~N}$ : (a) system trajectory in the space $\left(\varphi_{r}-\varphi_{b}, \dot{\varphi}_{r}, \dot{\varphi}_{b}\right), \bullet \mathbf{x}_{\text {in }}$, $\mathbf{x}_{\text {out }}, \boldsymbol{\hbar}$ quasi-equilibrium point $(\tilde{\mathbf{x}})$, (b) angular velocities, — bit angular velocity $\left(\dot{\varphi}_{b}\right),-$ - - top-rotary system velocity $\left(\dot{\varphi}_{r}\right)$.

with $\tilde{x}_{1}, \ldots, \tilde{x}_{n-3}$ as defined in Eqs. (28)-(30). The derivative of $V$ along the trajectories of $\dot{\mathbf{x}}=\mathbf{f}_{\mathbf{s}}\left(\mathbf{x}, W_{o b}, u\right)$ is:

- For $n=4$ :

$$
\dot{V}(\mathbf{x})=-\left(c_{t}+c_{r}\right) x_{2}^{2}
$$

- For $n=6$ :

$$
\dot{V}(\mathbf{x})=-c_{r} x_{2}^{2}-c_{t b} x_{4}^{2}-c_{t}\left(x_{2}-x_{4}\right)^{2}
$$

- For $n>6$ :

$$
\dot{V}(\mathbf{x})=-c_{r} x_{2}^{2}-c_{t b} x_{n-2}^{2}-c_{t}\left[\left(x_{2}-x_{4}\right)^{2}-\cdots-\left(x_{n-6}-x_{n-4}\right)^{2}\right]-c_{t l}\left(x_{n-4}-x_{n-2}\right)^{2} .
$$

Consequently, $\dot{V}(\mathbf{x}) \leqslant 0$. Due to the fact that $\dot{V}(\mathbf{x})=0$ only for $\mathbf{x}=\tilde{\mathbf{x}}$, by LaSalle's invariance principle [20], the quasi-equilibrium $\tilde{\mathbf{x}}$ is asymptotically stable.

From the form of the sliding region $\tilde{\Sigma}$, important system characteristics can be concluded, mainly, the ones referring to the stick-slip behaviour. First, the importance of the drill collars and the bit dynamics in the presence of a sliding motion. Second, the influence of $W_{o b}$ on bit sticking phenomena can be identified in the following way. Considering $u$ fixed and $W_{o b}$ as a varying parameter, for each $W_{o b}$, a different $\tilde{\Sigma}$ is obtained, consequently, a different periodic orbit is obtained. This fact is clearly noticed in Fig. 5(a) where different periodic orbits are obtained for each $W_{o b}$; in the figure, a fixed $u=8.1 \mathrm{Nm}$ is used. Furthermore, for a fixed $u$, when $W_{o b}$ is greater than a value, the bit is permanently stuck (Fig. 5(b)). In addition, the smaller $W_{o b}$ is, the smaller the sliding region $\tilde{\Sigma}$ is, and the trajectory is less likely to enter the sliding domain. This explains the fact that for a fixed $u$, reducing the $W_{o b}$ makes the stick-slip phenomenon disappear, and when $W_{o b}$ is too large the bit is completely stopped. This is accordance with drillers' experiences [1,11].

\subsection{Identification of parameter regions for non-desired phenomena: bifurcations classification}

Depending on variations of $W_{o b}$ and $u$, three kinds of dynamical behaviour can be identified in the system related to the switching manifold $\Sigma$ : (i) stick-slip at $\dot{\varphi}_{b}$, that is, the trajectory enters and leaves repeatedly the sliding mode, (ii) permanent bit stuck, that is, $\mathbf{x}(t) \in \tilde{\Sigma}, \forall t$, (iii) the bit moves in a constant velocity, in other 
(a)

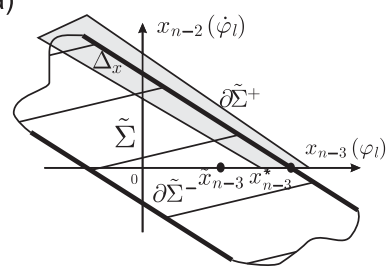

(b)

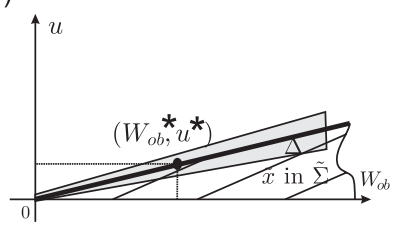

Fig. 6. Regions where stick-slip is possible (shadowed regions): (a) Region $\Delta_{x}$ in $\left(x_{n-3}, x_{n-2}\right)$ with $\mathbf{x} \in \Sigma,-\partial \tilde{\Sigma}^{+}, \partial \tilde{\Sigma}^{-}$(b) stick-slip region $\Delta$ in the $\left(W_{o b}, u\right)$ space, $-u^{+}=\mu_{s_{b}} R_{b} W_{o b}\left(\tilde{\mathbf{x}} \in \partial \tilde{\Sigma}^{+}\right)$. Wide-lined region corresponds to $\tilde{\Sigma}$. $\tilde{x}_{n-3}$ is the $(n-3)$ th component of the system quasi-equilibrium point, $x_{n-3}^{*}$ is the intersection of $\partial \tilde{\Sigma}^{+}$with $x_{n-2}=0,\left(W_{o b}^{*}, u^{*}\right)$ are reference values of $W_{o b}$ and $u$.

words, the trajectory moves towards an equilibrium outside $\Sigma$. Different regions in the state space and in the two-dimensional parameter space $\left(W_{o b}, u\right)$ can be identified through the form of the sliding manifold $\tilde{\Sigma}$.

In Fig. 6(a), region $\tilde{\Sigma}$ with the boundaries $\partial \tilde{\Sigma}^{+}$and $\partial \tilde{\Sigma}^{-}$is given. The study is focused on $\partial \tilde{\Sigma}^{+}$due to the fact that the points $\mathbf{x}_{\text {out }}$ through which the system trajectories go out of $\tilde{\Sigma}$ are near $\partial \tilde{\Sigma}^{+}$.

Two points are distinguished in Fig. 6(a) in the plane $\left(x_{n-3}, x_{n-2}\right)=\left(\varphi_{l}, \dot{\varphi}_{l}\right)$ : the $(n-3)$ th component of the quasi-equilibrium point, $\tilde{x}_{n-3}$, and $x_{n-3}^{*}$ denoting the intersection of $\partial \tilde{\Sigma}^{+}$with $x_{n-2}=0$, which has the following form:

$$
x_{n-3}^{*}= \begin{cases}\frac{\mu_{s_{b}} W_{o b} R_{b}}{k_{t}}+\bar{\varphi}_{b} & \text { if } n=4, \\ \frac{\mu_{s_{b}} W_{o b} R_{b}}{k_{t b}}+\bar{\varphi}_{b} & \text { if } n>4 .\end{cases}
$$

Note that if $n=4$, the study would be restricted to the plane $\left(\varphi_{r}, \dot{\varphi}_{r}\right)$.

Two key characteristics which determine transitions between different dynamical system behaviours are the fact that $\tilde{\mathbf{x}}$ is asymptotically stable and the change of $\tilde{\mathbf{x}}$ from real to virtual, or viceversa. The real or virtual characteristic of $\tilde{\mathbf{x}}$ can be identified by the distance between $\tilde{x}_{n-3}$ and $x_{n-3}^{*}$, which depends on the difference between $u$ and $T_{s_{b}}=\mu_{s_{b}} W_{o b} R_{b}$. When $\tilde{x}_{n-3}=x_{n-3}^{*}$, two reference values of $u$ and $W_{o b}$ are obtained:

$$
u^{*}=\mu_{s_{b}} W_{o b} R_{b}, \quad W_{o b}^{*}=\frac{u}{\mu_{s_{b}} R_{b}} .
$$

Consequently, the following relations can be established:

$$
\begin{aligned}
& \tilde{x} \in \tilde{\Sigma} \Rightarrow-\mu_{s_{b}} W_{o b} R_{b}<u<\mu_{s_{b}} W_{o b} R_{b}, \\
& \tilde{x} \in \partial \tilde{\Sigma}^{+} \Rightarrow u=\mu_{s_{b}} W_{o b} R_{b}, \\
& \tilde{x} \in \partial \tilde{\Sigma}^{-} \Rightarrow u=-\mu_{s_{b}} W_{o b} R_{b}, \\
& \tilde{x} \notin \tilde{\Sigma} \Rightarrow\left(u>\mu_{s_{b}} W_{o b} R_{b}\right) \vee\left(u<-\mu_{s_{b}} W_{o b} R_{b}\right) .
\end{aligned}
$$

A region in a neighbourhood of $\partial \tilde{\Sigma}^{+}$where stick-slip oscillations are present (see Fig. 6(a)) can be defined as:

$$
\Delta_{x}:=\left\{\mathbf{x} \in \Sigma: x_{n-3}^{*}-\eta^{-} \leqslant x_{n-3} \leqslant x_{n-3}^{*}+\eta^{+}\right\}
$$

for some $\eta^{+}, \eta^{-}>0$. The region identified in the plane $\left(x_{n-3}, x_{n-2}\right)$ can be translated into the two-dimensional parameter space $\left(W_{o b}, u\right)$ considering the relative position of $\tilde{x}_{n-3}$ with respect to the boundaries of $\tilde{\Sigma}$. The boundaries $\partial \tilde{\Sigma}^{+}, \partial \tilde{\Sigma}^{-}$are translated into the straight lines:

$$
u^{+}=\mu_{s_{b}} W_{o b} R_{b}, \quad u^{-}=-\mu_{s_{b}} W_{o b} R_{b},
$$

respectively. The region $\Delta \subset W_{o b} \times u$ where stick-slip is present is obtained (see Fig. 6(b)). It is concluded that:

- For a fixed value of $u$, there exist constants $\varepsilon^{-}>0$ and $\varepsilon^{+}>0$ such that:

(i) $W_{o b}>W_{o b}^{*}+\varepsilon^{+} \Rightarrow$ the bit is permanently stuck $(\mathbf{x}(t) \in \Sigma, \forall t)$. 


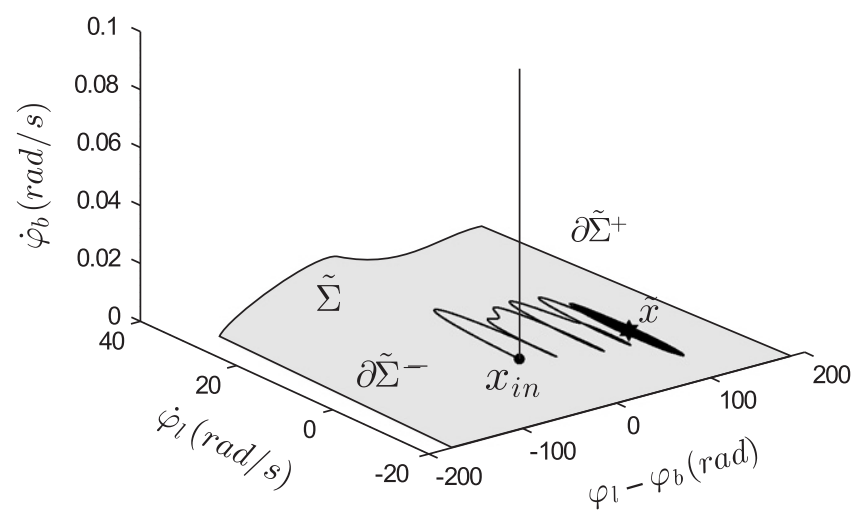

Fig. 7. Trajectory of the system in Eq. (3) with Eq. (13) with $n=8$ in the space $\left(\varphi_{l}-\varphi_{b}, \dot{\varphi}_{l}, \dot{\varphi}_{b}\right) . \tilde{\Sigma}$ sliding region (shadowed region), $\partial \tilde{\Sigma}^{+}$, $\partial \tilde{\Sigma}^{+}$boundaries of $\tilde{\Sigma}, \bullet \mathbf{x}_{\text {in }}, \star$ quasi-equilibrium point ( $\tilde{\mathbf{x}}$ ). Parameters in Eq. (14) are used with $W_{o b}=160 N, u=8.3 \mathrm{Nm}$. The trajectory of the system stays on $\tilde{\Sigma}$.

(a)

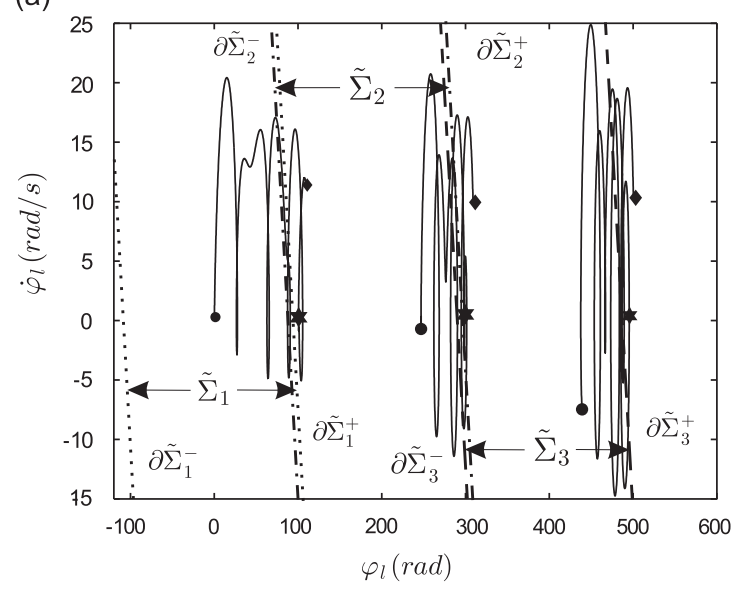

(b)

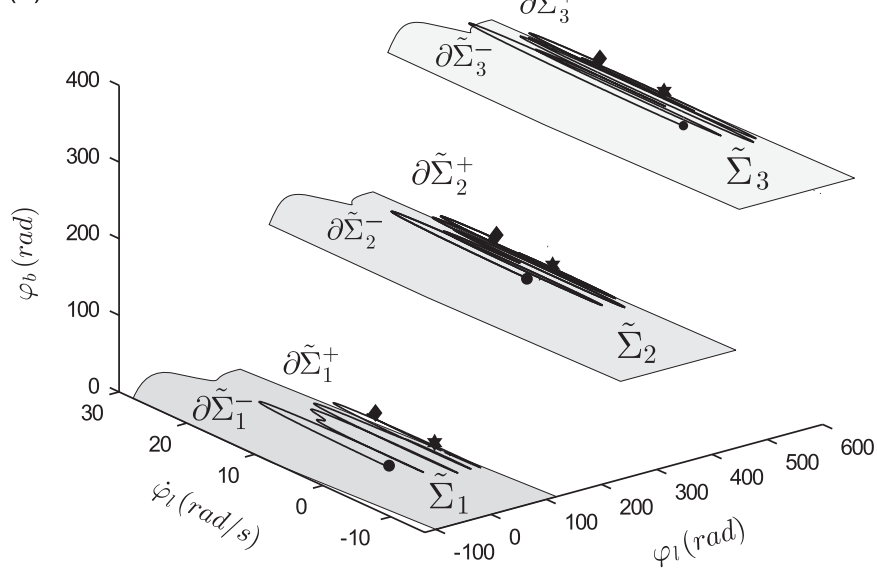

Fig. 8. Trajectory of the system in Eq. (3) with Eq. (13), with $n=8$ only for $x \in \tilde{\Sigma}$ : (a) projection of the trajectories in $\left(\varphi_{l}, \dot{\varphi}_{l}\right)$, (b) trajectories in the space $\left(\varphi_{l}, \dot{\varphi}_{l}, \varphi_{b}\right)$. Stick-slip is present and $\tilde{\Sigma}$ varies with time. The first three inputs to $\tilde{\Sigma}$ are considered. $\tilde{\Sigma}_{i}$ sliding region for the ith input to $\tilde{\Sigma}$ (shadowed region), $\partial \tilde{\Sigma}_{i}^{+}, \partial \tilde{\Sigma}_{i}^{+}$boundaries of $\tilde{\Sigma}_{i}, \bullet \mathbf{x}_{\text {in }}, \mathbf{x}_{\text {out }}, \star$ quasi-equilibrium point $(\tilde{\mathbf{x}}$ ).

(ii) $W_{o b}^{*}-\varepsilon^{-} \leqslant W_{o b} \leqslant W_{o b}^{*}+\varepsilon^{+} \Rightarrow$ stick-slip phenomenon at the bit.

(iii) $W_{o b}<W_{o b}^{*}-\varepsilon^{-} \Rightarrow$ the bit can move in a constant velocity $\left(\mathbf{x}(t)\right.$ can converge to $\left.\overline{\mathbf{x}}_{\mathbf{e}}\right)$.

- For a fixed value of $W_{o b}$, there exist constants $\rho^{-}>0$ and $\rho^{+}>0$ such that:

(i) $u<u^{*}-\rho^{-} \Rightarrow$ the bit is permanently stuck $(\mathbf{x}(t) \in \Sigma, \forall t)$.

(ii) $u^{*}-\rho^{-} \leqslant u \leqslant u^{*}+\rho^{+} \Rightarrow$ stick-slip phenomenon at the bit.

(iii) $u>u^{*}+\rho^{+} \Rightarrow \mathbf{x}(t)$ the bit can move in a constant velocity $\left(\mathbf{x}(t)\right.$ can converge to $\left.\overline{\mathbf{x}}_{\mathbf{e}}\right)$,

with $\overline{\mathbf{x}}_{\mathbf{e}}$ the standard equilibrium point of the system with positive rotary velocities. The smaller $W_{o b}^{*}$ and $u^{*}$ are, the smaller $\varepsilon^{+}, \varepsilon^{-}, \rho^{+}$and $\rho^{-}$are in order to have $\left(W_{o b}, u\right) \in \Delta$.

The types of dynamical behaviours described above have been identified in the system in Eq. (3) with Eq. (13). In Fig. 7, the situation of permanently stuck bit is depicted. Here, the system trajectory hits the sliding manifold only once and remains there, however, for other parameters, the system trajectory could hit $\tilde{\Sigma}$ several times before remaining on it.

Fig. 8 shows the stick-slip situation for $n=8$ (see Fig. 4). It must be pointed out that the smaller $W_{o b}$ is, the smaller $\tilde{\Sigma}$ is and the less time the system trajectories lie on $\tilde{\Sigma}$, i.e., the bit remains stuck during less time. $\tilde{\Sigma}$ 
(a)

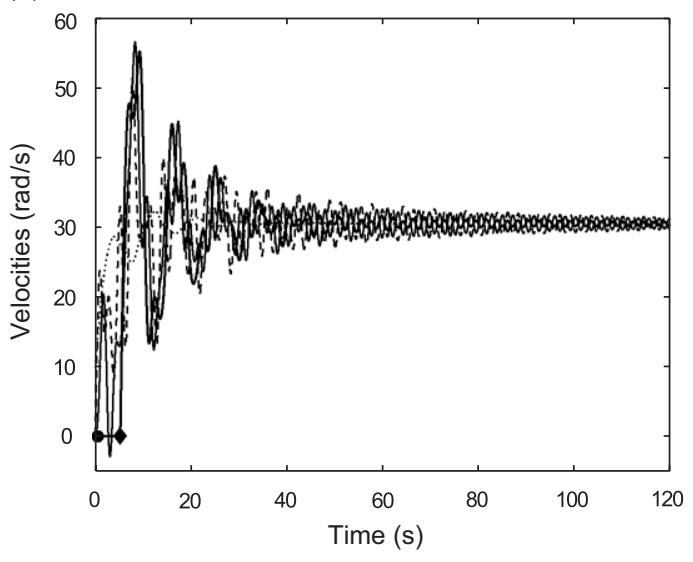

(b)

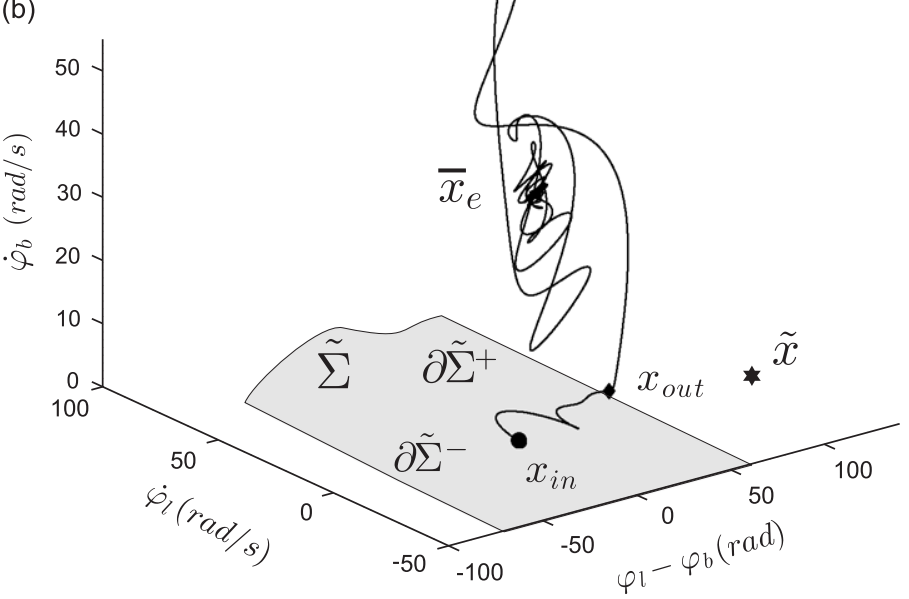

Fig. 9. Trajectory of the system in Eq. (3) with Eq. (13) with $n=8$. It approaches $\overline{\mathbf{x}}_{\mathbf{e}}$. Parameters in Eq. (14) are used with $W_{o b}=40 N$, $u=8.3 \mathrm{Nm}$ : (a) angular velocities, $-\dot{\varphi}_{b},-\dot{\varphi}_{l},--\dot{\varphi}_{1}, \cdots \dot{\varphi}_{r}$, (b) trajectory in the space $\left(\varphi_{l}-\varphi_{b}, \dot{\varphi}_{l}, \dot{\varphi}_{b}\right), \tilde{\Sigma}$ sliding region (shadowed region), $\partial \tilde{\Sigma}^{+}, \partial \tilde{\Sigma}^{+}$boundaries of $\tilde{\Sigma}, \bullet \mathbf{x}_{\text {in }}, \mathbf{x}_{\text {out }}, \boldsymbol{\star}$ quasi-equilibrium point $(\tilde{\mathbf{x}}), \overline{\mathbf{x}}_{\mathbf{e}}$ standard equilibrium point.

varies with time, due to the change of $x_{n-2}, x_{n-3}-x_{n-1}$ and $\bar{\varphi}_{b}$, which are the states defining the sliding manifold. Thus, $\tilde{\Sigma}=\bigcup_{i=1}^{n_{i n}} \tilde{\Sigma}_{i}$ with $n_{\text {in }}$ the number of times that system trajectory enters $\tilde{\Sigma}$.

Finally, Fig. 9 shows the desired situation when the drillstring is moving in a constant velocity. This is the situation when the system trajectory moves further away $\tilde{\Sigma}$ and approaches the equilibrium point $\left(\overline{\mathbf{x}}_{\mathbf{e}}\right)$. The local stability of $\overline{\mathbf{x}}_{\mathbf{e}}$ will be studied in Section 4 .

The three situations observed in simulations can be explained by means of relations given in Eq. (39) and the asymptotic stability of the quasi-equilibrium $\tilde{\mathbf{x}}$ :

- $\tilde{\mathbf{x}} \in \tilde{\Sigma}$ and $\tilde{\mathbf{x}}$ is far away enough from $\partial \tilde{\Sigma}^{+}$. If the system is in the sliding motion, then the system trajectories tend asymptotically to $\tilde{\mathbf{x}}$ and the bit is permanently stuck. Depending on the distance between $\tilde{\mathbf{x}}$ and $\partial \tilde{\Sigma}^{+}$, the trajectories may enter and leave $\tilde{\Sigma}$ several times before remaining on it.

- $\tilde{\mathbf{x}}$ is next to $\partial \tilde{\Sigma}^{+}$(it can be inside or outside $\tilde{\Sigma}$ ). If the system is in the sliding motion, then when system trajectories approach $\tilde{\mathbf{x}}$, they can leave the sliding domain and tend to a system equilibrium point with velocities greater than zero $\left(\overline{\mathbf{x}}_{\mathbf{e}}\right)$. However, if they do not reach it, they can fall again into the sliding domain. This is repeated continuously, and it gives rise to the stick-slip motion.

- $\tilde{\mathbf{x}} \notin \tilde{\Sigma}$ and it is far away enough from $\partial \tilde{\Sigma}^{+}$. If the system is in the sliding motion and the trajectories move towards $\tilde{\mathbf{x}}$, they can leave the sliding domain and fall into the domain of attraction of the system equilibrium point $\overline{\mathbf{x}}_{\mathbf{e}}$ for velocities greater than zero. As in the first situation, depending on the distance between $\tilde{\mathbf{x}}$ and $\partial \tilde{\Sigma}^{+}$, the trajectories may enter and leave $\tilde{\Sigma}$ several times before converging to $\overline{\mathbf{x}}_{\mathbf{e}}$.

To conclude with, a range of the weight of bit and the torque $u$ for which bit stick-slip or permanent stuck bit are present can be identified depending on drill collars dynamics, bit radius and bit-borehole friction characteristics.

\section{Assuring a desirable performance under fluctuating velocities and WOB by means of Hopf bifurcations analysis}

A desirable performance for the drillstring is that it moves in a constant positive velocity. In dynamical terms, this means that system trajectories approach a standard equilibrium point with velocities greater than zero. Therefore, the goal is to assure the stability of the system equilibrium under fluctuating parameters. In this section, the local stability of the system standard equilibrium and the presence of periodic orbits around it 
due to Hopf bifurcations are studied. Ranges of velocities at equilibrium and the weight on the bit for which Hopf bifurcations can appear will be identified.

\subsection{Preliminary definitions}

A new vector state will be defined in order to have a standard equilibrium point of the system with the same equilibrium velocities and the possibility of choosing them greater than zero. Let define,

$$
\varphi_{r, 1}=\varphi_{r}-\varphi_{1}, \quad \varphi_{j, j+1}=\varphi_{j}-\varphi_{j+1}, \quad \varphi_{p, l}=\varphi_{p}-\varphi_{l}, \quad \varphi_{l, b}=\varphi_{l}-\varphi_{b},
$$

with $j=1, \ldots, p-1$, and $p$ the number of drill pipes. The new vector state is:

$$
\begin{aligned}
\mathbf{x}^{\prime} & =\left(\dot{\varphi}_{r}, \varphi_{r, 1}, \ldots, \dot{\varphi}_{j}, \varphi_{j, j+1}, \ldots, \dot{\varphi}_{p}, \varphi_{p, l}, \dot{\varphi}_{l}, \varphi_{l, b}, \dot{\varphi}_{b}\right)^{\mathrm{T}} \\
& =\left(x_{1}^{\prime}, x_{2}^{\prime}, \ldots, x_{n-1}^{\prime}\right)^{\mathrm{T}} \in \mathbb{R}^{n-1},
\end{aligned}
$$

where $n$ is the dimension of the system in Eq. (3). Let consider $\dot{\varphi}_{b}=x_{n-1}^{\prime}>0$. The system is now rewritten as:

$$
\dot{\mathbf{x}}^{\prime}(t)=\mathbf{A}^{\prime} \mathbf{x}^{\prime}(t)+\mathbf{B}^{\prime} u+\mathbf{T}_{\mathbf{f}}^{\prime}\left(\mathbf{x}^{\prime}(t)\right)
$$

with:

$$
\mathbf{B}^{\prime}=\left(\begin{array}{c}
1 \\
J_{r} \\
0 \\
\vdots \\
0
\end{array}\right), \quad \mathbf{T}_{\mathbf{f}}^{\prime}\left(\mathbf{x}^{\prime}\right)=\left(\begin{array}{c}
0 \\
\vdots \\
0 \\
-\frac{T_{f_{b}}^{+}\left(x_{n-1}^{\prime}\right)}{J_{b}}
\end{array}\right)
$$

and $\mathbf{A}^{\prime}$ a constant matrix depending on system physical parameters. The dimension of the system in Eq. (43) will be denoted by $n^{\prime}$, therefore, $\mathbf{x}^{\prime} \in \mathbb{R}^{n^{\prime}}$ with $n^{\prime}=n-1$. Note that $n^{\prime}$ is an odd integer.

Let $\Omega>0$ be the rotary velocity at equilibrium. The system in Eq. (43) has an unique equilibrium point $\overline{\mathbf{x}_{\mathbf{e}}^{\prime}}$ for each fixed $\Omega$ which is the solution of the set of equations:

$$
\begin{aligned}
& \dot{\varphi}_{r}=\dot{\varphi}_{1}=\ldots \dot{\varphi}_{p}=\dot{\varphi}_{l}=\dot{\varphi}_{b}=\Omega, \Omega>0, \\
& u-\left(c_{r}+c_{b}\right) \Omega-\bar{T}_{f_{b}}^{+}(\Omega)=0, \\
& h(\Omega)=c_{b} \Omega+\bar{T}_{f_{b}}^{+}(\Omega), \\
& \bar{\varphi}_{r, 1}=\bar{\varphi}_{j, j+1}=\frac{h(\Omega)}{k_{t}}, \quad j=1, \ldots, p-1, \\
& \bar{\varphi}_{p, l}=\frac{h(\Omega)}{k_{t l}}, \quad \bar{\varphi}_{l, b}=\frac{h(\Omega)}{k_{t b}},
\end{aligned}
$$

with

$$
\begin{aligned}
& u>\mu_{s_{b}} R_{b} W_{o b}, \quad \bar{T}_{f_{b}}^{+}(\Omega)>0, \quad \forall \Omega>0, \\
& \bar{T}_{f_{b}}^{+}(\Omega)=W_{o b} R_{b}\left[\mu_{c_{b}}+\left(\mu_{s_{b}}-\mu_{c_{b}}\right) \mathrm{e}^{-\gamma_{b} / v_{f} \Omega}\right] .
\end{aligned}
$$

Let consider a system of the form

$$
\dot{\mathbf{x}}=\mathbf{f}(\mathbf{x}, \mu),
$$

where $\mathbf{f}: \mathbb{R}^{n^{\prime}} \times \mathbb{R} \rightarrow \mathbb{R}^{n^{\prime}}$ is a smooth mapping, $\mathbf{x} \in \mathbb{R}^{n^{\prime}}$ and $\mu \in \mathbb{R}$.

Definition 4 (Hale and Koçak [21], Liu [22], Seydel [23]). A system in Eq. (47) with an isolated equilibrium point $\overline{\mathbf{x}_{\mathbf{e}}^{\prime}}$ undergoes a Hopf bifurcation $(\mathrm{HB})$ at $\overline{\mathbf{x}_{\mathbf{e}}^{\prime}}$ when a simple pair of complex conjugate eigenvalues of the Jacobian $\mathbf{J}\left(\overline{\mathbf{x}_{\mathbf{e}}^{\prime}}, \mu\right)$ crosses the imaginary axis from left to right, while all other eigenvalues have negative real parts. 
Theorem 5 (Liu [22]). A system in Eq. (47) with an isolated equilibrium point $\overline{\mathbf{x}}_{\mathbf{e}}^{\prime}$ undergoes a Hopf bifurcation for $\mu=\bar{\mu}$ if:

$$
\Delta_{1}(\bar{\mu})>0, \quad \Delta_{2}(\bar{\mu}), \ldots, \Delta_{n^{\prime}-2}(\bar{\mu})>0, \quad a_{0}(\bar{\mu})>0, \quad \Delta_{n^{\prime}-1}=0
$$

and

$$
\left.\frac{\mathrm{d} \Delta_{n^{\prime}-1}(\mu)}{\mathrm{d} \mu}\right|_{\mu=\bar{\mu}} \neq 0,
$$

where $\Delta_{i}(\bar{\mu})$ stands for the Hurwitz determinants of order $i$ of the characteristic polynomial of $\mathbf{J}\left(\overline{\mathbf{x}^{\prime}} \mathbf{e}, \bar{\mu}\right)$ and $a_{0}(\bar{\mu})$ is the zero-order term of this polynomial. The condition in Eq. (49) is referred to as transversality condition. Under conditions in Eqs. (48) and (49), in any neighbourhood $\mathscr{U}$ of $\overline{\mathbf{x}^{\prime}}$ and any given $\mu_{0}>\bar{\mu}$ there is a $\mu^{*}$ with $\left|\mu^{*}\right|<\mu_{0}$ such that the system has a periodic orbit in $\mathscr{U}$.

\subsection{Presence of Hopf bifurcation points for the system of two degrees of freedom}

From Theorem 5, the following proposition can be established. It defines the region of angular velocities $\Omega$ and parameter $W_{o b}$ for which Hopf bifurcations are present in the drillstring for $n^{\prime}=3$. Let consider the characteristic polynomial of the Jacobian matrix of the system in Eq. (43) at the equilibrium point $\overline{\mathbf{x}_{\mathbf{e}}^{\prime}}=$ $\left(\Omega, \bar{\varphi}_{r, b}, \Omega\right)$ as:

$$
\lambda^{3}+a_{2} \lambda^{2}+a_{1} \lambda+a_{0}=0,
$$

with:

$$
\begin{aligned}
& a_{2}\left(\Omega, W_{o b}\right)=\frac{1}{J_{b} J_{r}}\left[J_{r}\left(c_{b}+c_{t}+d_{b}(\Omega)\right)+J_{b}\left(c_{r}+c_{t}\right)\right], \\
& a_{1}\left(\Omega, W_{o b}\right)=\frac{1}{J_{b} J_{r}}\left[c_{b}\left(c_{r}+c_{t}\right)+c_{t}\left(c_{r}+d_{b}(\Omega)\right)+d_{b}(\Omega) c_{r}+k_{t}\left(J_{b}+J_{r}\right)\right], \\
& a_{0}\left(\Omega, W_{o b}\right)=\frac{k_{t}}{J_{b} J_{r}}\left(c_{b}+c_{r}+d_{b}(\Omega)\right), \\
& d_{b}(\Omega)=-W_{o b} R_{b} \frac{\gamma_{b}}{v_{f}}\left(\mu_{s_{b}}-\mu_{c_{b}}\right) \mathrm{e}^{-\left(\gamma_{b} / v_{f}\right) \Omega} .
\end{aligned}
$$

Proposition 6. Consider a system in Eq. (43) with the vector state $\mathbf{x}^{\prime}=\left(\dot{\varphi}_{r}, \varphi_{r, b}, \dot{\varphi}_{b}\right), \dot{\varphi}_{r}>0, \dot{\varphi}_{b}>0$ and the equilibrium point $\overline{\mathbf{x}}_{\mathbf{e}}^{\prime}=\left(\Omega, \bar{\varphi}_{r, b}, \Omega\right)$ with $\Omega \in\left(0, \Omega_{f}\right]$ and $\Omega_{f}>0$. Consider the characteristic polynomial of Eq. (50). Let $\Omega, W_{o b} \in \mathbb{R}^{+}-\{0\} \quad$ and $\quad \Delta_{2}\left(\Omega, W_{o b}\right)=a_{1}\left(\Omega, W_{o b}\right) \quad a_{2}\left(\Omega, W_{o b}\right)-a_{0}\left(\Omega, W_{o b}\right), \quad \mathscr{H}_{+}:=\left\{\Omega \times W_{o b}\right.$ : $\left.a_{0}\left(\Omega, W_{o b}\right)>0, a_{1}\left(\Omega, W_{o b}\right)>0\right\}$ and $\mathscr{H}_{0}:=\left\{\Omega \times W_{o b}: \Delta_{2}\left(\Omega, W_{o b}\right)=0\right\}$. Then, for $\Omega \times W_{o b} \in \mathscr{H}_{+} \cap \mathscr{H}_{0}$ the system undergoes a Hopf bifurcation.

Proof. Conditions in Eqs. (48) and (49) of Theorem 5 will be checked. Considering $\Omega$ as a varying velocity, relation $\Delta_{2}=0$ yields to $\overline{W_{o b} 1,2}$ :

$$
\overline{W_{o b 1,2}}=\frac{v_{f} \mathrm{e}^{\left(\gamma_{b} \Omega / v_{f}\right)}}{2 \gamma_{b} R_{b} J_{r}\left(\mu_{s_{b}}-\mu_{c_{b}}\right)\left(c_{r}+c_{t}\right)}[\mathscr{A} \pm \sqrt{\mathscr{B}}],
$$

where $\mathscr{A}, \mathscr{B}$ depend on physical parameters.

The fact that $\left(\Omega, W_{o b}\right) \in \mathscr{H}_{+}$assures the complex eigenvalues to move from left (stability region) to right (instability region) of the imaginary axis as $W_{o b}$ and $\Omega$ are varied.

The condition in Eq. (49) must be also accomplished. For the system in Eq. (43), it takes the form:

$$
\left.\frac{\mathrm{d} \Delta_{2}\left(\Omega, W_{o b}\right)}{\mathrm{d} W_{o b}}\right|_{W_{o b}=\overline{W_{o b} 1,2}}= \pm \frac{1}{J_{b}^{2} J_{r}^{2} v_{f}^{2}}\left[\gamma_{b} R_{b}\left(\mu_{c_{b}}-\mu_{s_{b}}\right) \mathrm{e}^{-\gamma_{b} \Omega / v_{f}} \sqrt{\mathscr{\mathscr { B }}}\right] \neq 0 .
$$

The condition in Eq. (52) is met for $\mathscr{B}>0$, which is the condition for $\overline{W_{o b}} 1,2$ to be real. Due to the exponential function in Eq. (52), function $\mathrm{d} \Delta_{2}\left(\Omega, W_{o b}\right) /\left.\mathrm{d} W_{o b}\right|_{W_{o b}}=\overline{W_{o b} 1,2}$ approaches zero as $\Omega$ approaches infinity. As $\Omega$ is bounded by $\Omega_{f}$ from above, Eq. (52) will never equal zero.

In conclusion, a Hopf bifurcation appears for the set of values $\left(\Omega, W_{o b}\right) \in \mathscr{H}_{+} \cap \mathscr{H}_{0}$. 
(a)

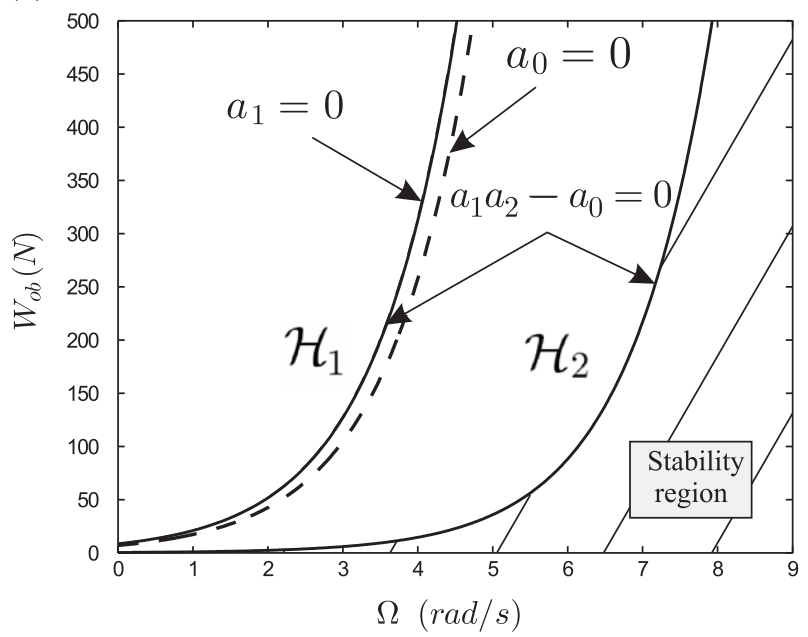

(b)

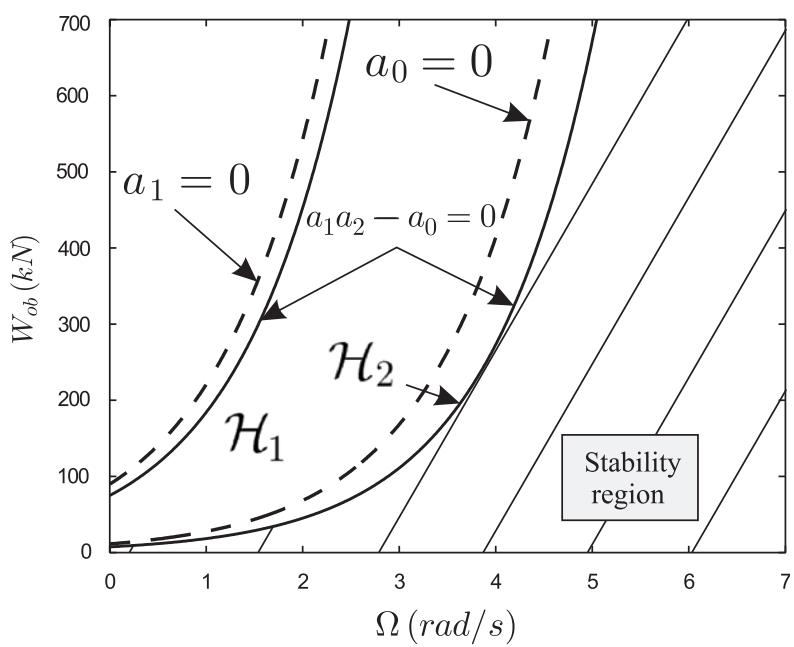

Fig. 10. $\left(\Omega, W_{o b}\right)$ for which locally stability of the equilibrium in Eq. (45) is met (wide-lined region): (a) Parameters in Eq. (14) are used, (b) parameters in Eq. (53) of a real drillstring are used. $\mathscr{H}_{1}, \mathscr{H}_{2}$ curves obtained making $\Delta_{2}=0$. Curve $\mathscr{H}_{2}$ is the locus of Hopf bifurcation.

The bifurcation behaviour of the system with $n^{\prime}=3$ will be studied through the conditions of Proposition 6 for parameters in Eq. (14) and for more realistic parameters, taken from a real drillstring. The following drillstring design is used [26]: (i) a drill pipe line consisting of one hundred pipe lines of 5 inches of outer diameter (OD), 4.408 in of inner diameter (ID) and $9 \mathrm{~m}$ of length; (ii) two units of drill collars of $6^{\frac{1}{2}}$ in and $18.60 \mathrm{~m}$ of length; (iii) stabilizers of $6^{\frac{1}{2}}$ (ID), $12^{\frac{1}{4}}$ (OD) and $1.5 \mathrm{~m}$ of length; (iv) a heavy-weight drill pipe of $6^{\frac{1}{2}}$ of diameter and $9.30 \mathrm{~m}$ of length; (v) a roller-cone bit of $6^{\frac{2}{2}}$ (ID), $12^{\overline{4}}$ (OD), $1.5 \mathrm{~m}$ of length. Thus:

$$
\begin{aligned}
& J_{r}=2122 \mathrm{~kg} \mathrm{~m}^{2}, \quad J_{b}=471.9698 \mathrm{~kg} \mathrm{~m}^{2}, \quad R_{b}=0.155575 \mathrm{~m}, \quad k_{t}=861.5336 \mathrm{Nm} / \mathrm{rad}, \\
& c_{t}=172.3067 \mathrm{Nm} \mathrm{s} / \mathrm{rad}, \quad c_{r}=425 \mathrm{Nm} \mathrm{s} / \mathrm{rad}, \quad c_{b}=50 \mathrm{Nm} \mathrm{s} / \mathrm{rad}, \\
& \mu_{c_{b}}=0.5, \quad \mu_{s_{b}}=0.8, \quad D_{v}=10^{-6}, \gamma_{b}=0.9, \quad v_{f}=1 .
\end{aligned}
$$

For parameters in Eqs. (14) and (53), Fig. 10 depicts the curves $\mathscr{H}_{1}$ and $\mathscr{H}_{2}$, obtained making $\Delta_{2}=0$. The curve $\mathscr{H}_{+} \cap \mathscr{H}_{0}=\mathscr{H}_{2}$ is a locus of Hopf bifurcation points referred to as the Hopf bifurcation boundary. The stability region of equilibrium point $\overline{\mathbf{x}^{\prime}}$ e is stated as the region below $\mathscr{H}_{2}$ in which $a_{0}>0, a_{1}>0$ and $\Delta_{2}>0$.

Bifurcation points must be avoided in order to have a desired performance of the system (no oscillations). From Fig. 10, safe ranges of $W_{o b}$ and $\Omega$ can be established. In order to drive angular velocities at the equilibrium $(\Omega)$ to a desired value, a control mechanism can be added at the top-rotary system in order to maintain $\dot{\varphi}_{r}$ to a desired value, and in consequence, the rest of velocities at the equilibrium (see Refs. [2,24]).

\subsection{Multiple Hopf bifurcations for the generic model}

Multiple Hopf bifurcations may occur in the system in Eq. (43) in a neighbourhood of the equilibrium point in Eq. (45) for $n^{\prime}>3$. In other words, an arbitrary number $s$ of pairs of pure imaginary eigenvalues $\pm \mathrm{i} \omega_{1}, \ldots, \pm \mathrm{i} \omega_{s}$ with $\omega_{j}>0, \forall j$ may appear. Then, periodic and quasi-periodic motions are likely to happen.

Different methods for the identification of multiple Hopf bifurcations have been proposed, for example, see Ref. [25]. For the drillstring, these methods become untractable. This section proposes the prevention of these bifurcations by avoiding the crossings of multiple complex conjugate eigenvalues through the imaginary axis.

Let consider the characteristic equation of the linearized system in Eq. (43) around equilibrium $\overline{\mathbf{x}_{\mathbf{e}}^{\prime}}$ :

$$
\lambda^{n^{\prime}}+a_{n^{\prime}-1} \lambda^{n^{\prime}-1}+\cdots+a_{1} \lambda+a_{0}=0
$$


(a)

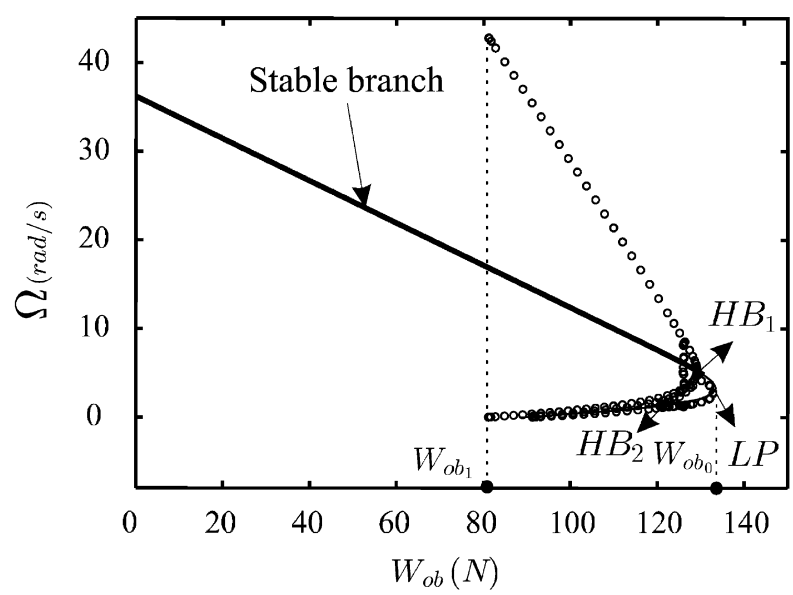

(c)

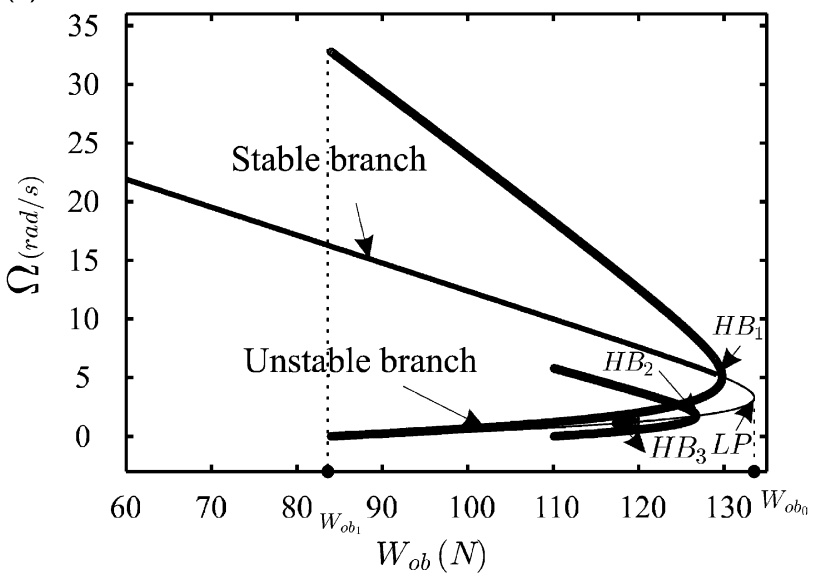

(b)

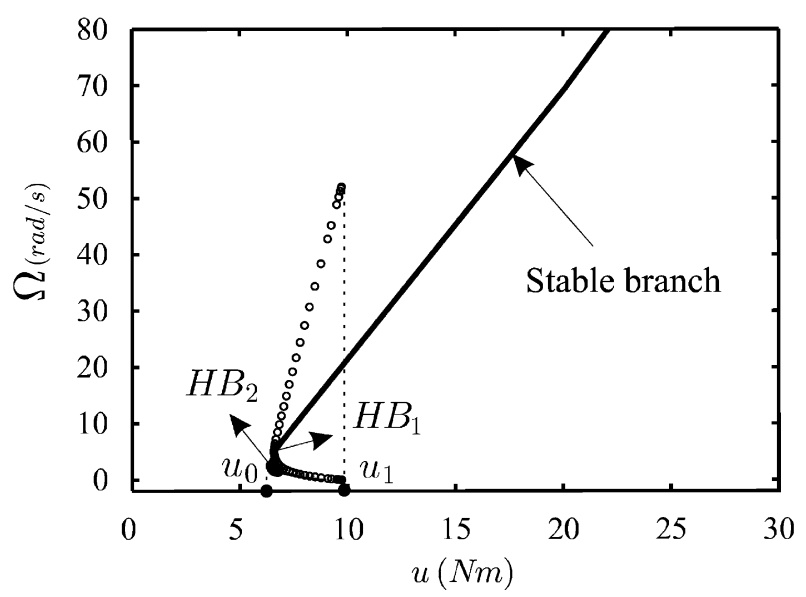

(d)

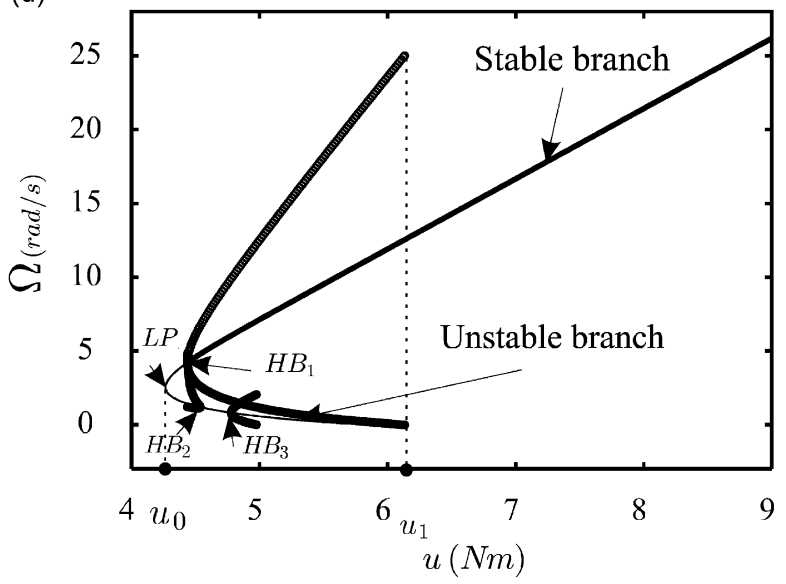

Fig. 11. Bifurcation diagrams varying $W_{o b}$ and $u$, for the system in Eq. (43) for $\Omega>0$ and parameters in Eq. (14): (a) $n^{\prime}=3, u=8.1 \mathrm{Nm}$, (b) $n^{\prime}=3, W_{o b}=80 \mathrm{~N}$, (c) $n^{\prime}=7, u=8.1 \mathrm{Nm}$, (d) $n^{\prime}=7, W_{o b}=60 \mathrm{~N}$. — stable branch, - unstable branch, o periodic orbits, $H B_{i}$ Hopf bifurcation points, $L P$ turning points.

The solutions of Eq. (54) would be of the form $\lambda= \pm \mathrm{i} \omega_{j}, \lambda=\alpha \in \mathbb{R}$, with $j=1, \ldots,\left(n^{\prime}-1\right) / 2$ the number of pairs of pure imaginary eigenvalues, if the following relations were met:

$$
a_{l}-a_{n^{\prime}-1} a_{k}=0, \quad l=\left(n^{\prime}-1\right)-2 j, \quad k=n^{\prime}-2 j, j=1, \ldots, \frac{n^{\prime}-1}{2} .
$$

Then, $\left(n^{\prime}-1\right) / 2$ relations are obtained. Each of them establishes a second-order equation in $W_{o b}$ depending on $\Omega$. The values of $W_{o b}$ and $\Omega$ satisfying these relations should be avoided.

The region in which no Hopf bifurcation is present for the system with $n^{\prime}=3$ is a good estimation for this region in case $n^{\prime}>3$. This can be verified by means of the bifurcation diagrams with respect to parameters $W_{o b}$ and $u$ depicted in Fig. 11 for the system in Eq. (43) in the cases $n^{\prime}=3$ and $n^{\prime}=7$. The bifurcation diagrams have been obtained by means of AUTO tool within XPPAUT [27].

In the bifurcation diagrams, a stable (the thickest one) and an unstable branches are given. The stable branch represents the values of $\left(W_{o b}, \Omega\right)$ or $(u, \Omega)$ for which the system converges to an equilibrium point; whereas the unstable branch represents the values of the parameters for which the system has an unstable equilibrium point. Two kinds of bifurcation points are given in the figures: (i) turning points (LP), (ii) Hopf bifurcation points $(H B)$. Periodic orbits are represented by circles, they emanate from Hopf bifurcation points.

Let consider the bifurcation diagram $\left(W_{o b}, \Omega\right)$, and let $W_{o b_{0}}$ and $W_{o b_{1}}$ be the values of $W_{o b}$ corresponding to the turning point of the unstable branch and the end point of the largest branch of periodic orbits, 
(a)

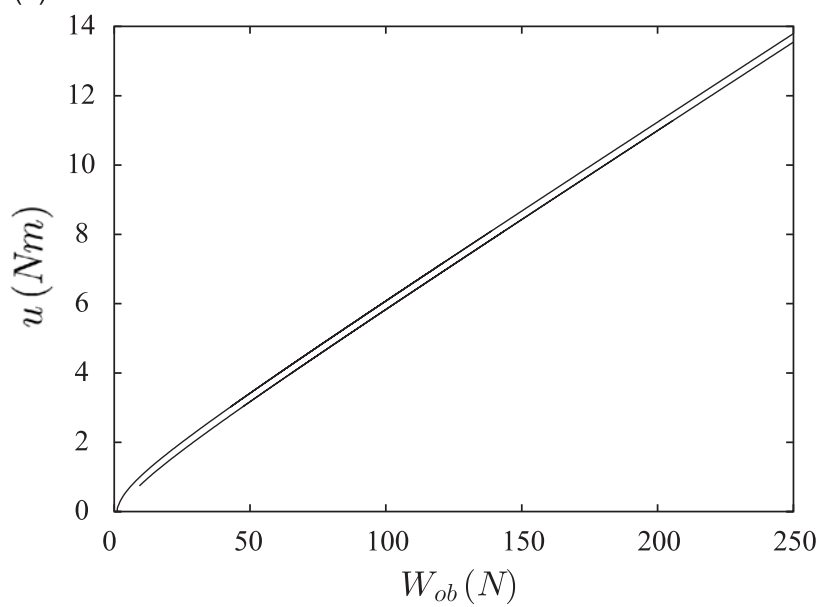

(b)

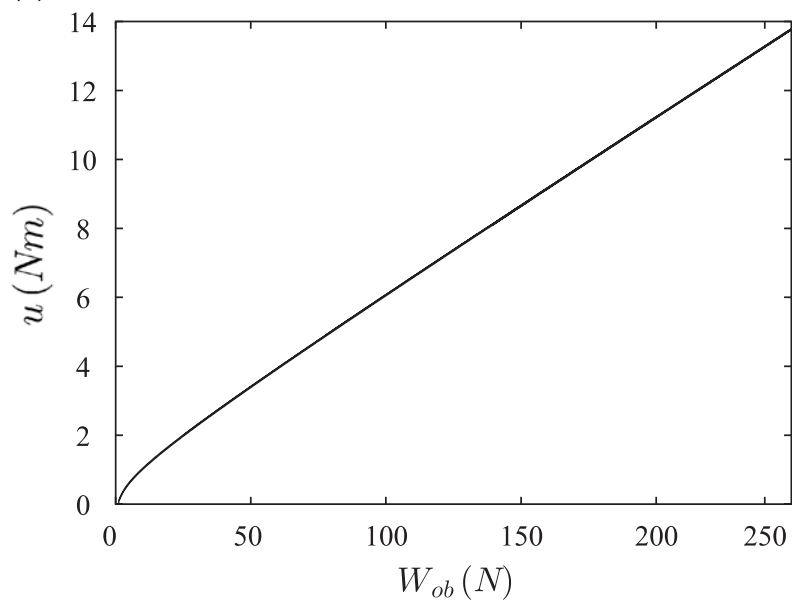

Fig. 12. Values $\left(W_{o b}, u\right)$ at which a Hopf bifurcation point can appear for the system in (43) with parameters in (14): (a) $n^{\prime}=3$, (b) $n^{\prime}=7$.

respectively. For values $W_{o b_{1}} \leqslant W_{o b} \leqslant W_{o b_{0}}$, the system may present oscillations. Notice that bifurcation diagrams for varying $W_{o b}$ 's are obtained for a fixed $u$. If a different value of $u$ were used, different values of $\left(W_{o b}, \Omega\right)$ at which Hopf bifurcation points are located would be obtained, and consequently, different values of $W_{o b_{0}}$ and $W_{o b_{1}}$. This is confirmed by Fig. 12 where the values $\left(W_{o b}, u\right)$ at which a Hopf bifurcation point is present are depicted. The graphics have been also obtained by means of AUTO tool within XPPAUT [27]. The same conclusions can be given for bifurcation diagrams $(u, \Omega)$. See Figs. 11(b) and (d), here, $u_{0}$ and $u_{1}$ are the values of $u$ corresponding to the turning point of the unstable branch and the end point of the largest branch of periodic orbits, respectively. The interval $u_{0} \leqslant u \leqslant u_{1}$ is a non-safe operation range for a fixed $W_{o b}$. Notice that $u_{0}$ and $u_{1}$ are different for $n^{\prime}=3$ and $n^{\prime}=7$ due to the fact that the bifurcation diagrams have been obtained for a different fixed value of $W_{o b}$ in each case.

To conclude with, the drillstring has a desired performance, that is, it moves in a constant velocity when: (i) for a fixed $u, W_{o b}$ is small enough, (ii) for a fixed $W_{o b}, u$ is high enough. This is accordance with results given in Section 3, indeed, comparing Figs. 12 and 6(b), it can be seen that region $\Delta$ where stick-slip is present intersects the values $\left(W_{o b}, u\right)$ where a Hopf bifurcation point may appear.

The identified ranges of $W_{o b}$, the torque supplied by the top-rotary mechanism $(u)$ and the rotary speed for which the drillstring has a desired performance are in accordance with field drillers' experiences [1,5,6,26]. Then, by means of the proposed analysis method of identifying bit sticking phenomena (Section 3) and oscillations around a constant velocity (Section 4), it is possible to estimate the problematic parameter ranges for a specific drillstring design.

\section{Conclusions}

Different bifurcations have been identified in an $n$-dimensional model of a conventional oilwell drillstring. Stick-slip oscillations at the bit have been shown to be related to the presence of a discontinuous dry friction modelling the bit-rock contact. The drillstring has been considered as a piecewise-smooth system and a sliding motion has been shown to be present. Ranges of key parameters in any drilling operation, such as, the weight on the bit $\left(W_{o b}\right)$, the motor torque given by the top-rotary mechanism $(u)$ and the rotary speed have been identified lest non-desired oscillatory phenomena appear in the system. Changes in drillstring behaviour have been interpreted as different bifurcations types. Not only system parameters changes have been shown to play an important role in the bifurcation behaviour, but also the characteristics of system equilibria.

Based on the analysis carried out, control methodologies can be designed in order to maintain system parameters within the proposed safe operation ranges. Finally, the analysis proposed can be applied to other mechanical systems exhibiting stick-slip oscillations which are described by similar models to the one studied in this work. 


\section{Acknowledgements}

The authors gratefully acknowledge the valuable discussions and support received by Professor Rodolfo Suárez Cortez in developing the first ideas of this work. They are also gratetul to Julien Cabillic (Institut Franç ais de Mécanique Avancée) who revised the drillstring mechanical model. This paper has been partially done in the context of LAFMAA project "VIBSARTAS" under CONACYT grant and under Ramón y Cajal contract under the support of the Ministerio de Educación y Ciencia (MEC) of Spain.

\section{References}

[1] J.F. Brett, The genesis of torsional drillstring vibrations. SPE Drilling Engineering (1992) 168-174.

[2] E.M. Navarro-López, R. Suárez, Mechanical vibrations in an oilwell drillstring: control problems, Iberoamerican Journal of Automatic Control and Industrial Computer Science 2 (1) (2005) 43-54 (in Spanish).

[3] S.L. Chen, K. Blackwood, E. Lamine, Field investigation of the effects of stick-slip, lateral and whirl vibrations on roller-cone bit performance, SPE Drilling and Completion 17 (1) (2002) 15-20.

[4] A.P. Christoforou, A.S. Yigit, Fully coupled vibrations of actively controlled drillstrings, Journal of Sound and Vibration 267 (2003) 1029-1045.

[5] Å. Kyllingstad, G.W. Halsey, A study of slip/stick motion of the bit, SPE Drilling Engineering (1988) 369-373.

[6] Y.-Q. Lin, Y.-H. Wang, Stick-slip vibration of drill strings, Journal of Engineering for Industry 113 (1991) $38-43$.

[7] F. Abbassian, V.A. Dunayevsky, Application of stability approach to torsional and lateral bit dynamics, SPE Drilling and Completion 13 (2) (1998) 99-107.

[8] J.D. Jansen, L. van den Steen, Active damping of self-excited torsional vibrations in oil well drillstrings, Journal of Sound and Vibration 179 (4) (1995) 647-668.

[9] N. Mihajlović, A.A. van Veggel, N. van de Wouw, H. Nijmeijer, Analysis of friction-induced limit cycling in an experimental drillstring system, ASME Journal of Dynamic Systems, Measurement and Control 126 (2004) 709-720.

[10] A.F.A. Serrarens, M.J.G. van de Molengraft, J.J. Kok, L. van den Steen, $H_{\infty}$ control for suppressing stick-slip in oil well drillstrings, IEEE Control Systems (1998) 19-30.

[11] P. Sananikone, O. Kamoshima, D.B. White, A field method for controlling drillstring torsional vibrations, Proceedings of the IADC/ SPE Drilling Conference, New Orleans, Louisiana, 1992, pp. 443-452.

[12] J.P. Maratier, Optimum Rotary Speed and Bit Weight for Rotary Drilling, MSc Thesis, Louisiana State University, 1971.

[13] B. Armstrong-Hélouvry, P. Dupont, C. Canudas de Wit, A survey of models, analysis tools, and compensation methods for the control of machines with friction, Automatica 30 (7) (1994) 1083-1183.

[14] R.I. Leine, D.H. van Campen, A. de Kraker, L. van den Steen, Stick-slip vibrations induced by alternate friction models, Nonlinear Dynamics 16 (1998) 41-54.

[15] D. Karnopp, Computer simulation of stick-slip friction in mechanical dynamic systems, ASME Journal of Dynamics Systems, Measurement, and Control 107 (1) (1985) 100-103.

[16] D.R. Pavone, J.P. Desplans, Application of high sampling rate downhole measurements for analysis and cure of stick-slip in drilling, Proceedings of the SPE Annual Technical Conference and Exhibition, New Orleans, Louisiana, 1994, pp. 335-345.

[17] V.I. Utkin, Sliding Modes in Control Optimization, Springer, Berlin, 1992.

[18] A.F. Filippov, Differential Equations with Discontinuous Right-hand Sides, Kluwer Academic Publishers, Dordrecht, 1988.

[19] F.B. Cunha, D.J. Pagano, U.F. Moreno, Sliding bifurcations of equilibria in planar variable structure systems, IEEE Transactions on Circuits and Systems-I: Fundamental Theory and Applications 50 (8) (2003) 1129-1134.

[20] J.P. LaSalle, The Stability of Dynamical Systems, Society for Industrial and Applied Mathematics, Hamilton Press, 1976.

[21] J. Hale, H. Koçak, Dynamics and Bifurcations, Springer, New York, 1991.

[22] W.M. Liu, Criterion of Hopf bifurcations without using eigenvalues, Journal of Mathematical Analysis and Applications 182 (1994) 250-256.

[23] R. Seydel, Practical Bifurcation and Stability Analysis, Springer, New York, 1994.

[24] E.M. Navarro-López, R. Suárez, Practical approach to modelling and controlling stick-slip oscillations in oilwell drillstrings, Proceedings of the IEEE Conference on Control Applications, IEEE Press, Taipei, Taiwan, 2004, pp. 1454-1460.

[25] W. Govaerts, J. Guckenheimer, A. Khibnik, Defining functions for multiple Hopf bifurcations, SIAM Journal on Numerical Analysis 34 (3) (1997) 1269-1288.

[26] Tri-Cone Manual de Barrenas, Hughes Tool Company, U.S.A., 1982.

[27] B. Ermentrout, Simulating, Analyzing, and Animating Dynamical Systems (A Guide to XPPAUT for Researchers and Students), SIAM Software Environments Tools, 2002. 